\title{
Modified Patch-based Locally Optimal Wiener Method for Interferometric SAR Phase Filtering
}

\author{
Yang Wang ${ }^{\mathrm{a}, *}$, Haifeng Huang ${ }^{\mathrm{a}}$, Zhen Dong ${ }^{\mathrm{a}}$, Manqing $\mathrm{Wu}^{\mathrm{a}, \mathrm{b}}$ \\ ${ }^{a}$ School of Electronic Science and Engineering, National University of Defense Technology, Changsha, 410073, \\ Hunan, China \\ ${ }^{\mathrm{b}}$ China Academy of Electronics and Information Technology, China Electronics Technology Group Corporation, \\ Beijing, 100000, China
}

\begin{abstract}
This paper presents a modified patch-based locally optimal Wiener (PLOW) method for interferometric synthetic aperture radar (InSAR) phase filtering. PLOW is a linear minimum mean squared error (LMMSE) estimator based on a Gaussian additive noise condition. It jointly estimates moments, including mean and covariance, using a non-local technique. By using similarities between image patches, this method can effectively filter noise while preserving details. When applied to InSAR phase filtering, three modifications are proposed based on spatial variant noise. First, pixels are adaptively clustered according to their coherence magnitudes. Second, rather than a global estimator, a locally adaptive estimator is used to estimate noise covariance. Third, using the coherence magnitudes as weights, the mean of each cluster is estimated, using a weighted mean to further reduce noise. The performance of the proposed method is experimentally verified using simulated and real data. The results of our study demonstrate that the proposed method is on par or better than the non-local interferometric SAR (NL-InSAR) method.
\end{abstract}

Keywords: Interferometric synthetic aperture radar (InSAR); Phase filtering; Patch-based locally optimal Wiener (PLOW); Linear minimum mean squared error (LMMSE); Coherence magnitude

\section{Introduction}

Synthetic aperture radar (SAR) is a very successful remote sensing technology with many applications. One of these applications is interferometry [1]. This technique is based on the generation of an interferogram using two complex images of the same scene taken from slightly different view angles. After generating an interferometric phase, phase unwrapping is used to obtain the final product, a digital elevation model (DEM). Affected by many decorrelation factors [1], [2] (such as temporal decorrelation, spatial decorrelation, volume decorrelation etc.), interferometric phase is disturbed by strong noise which handicaps the unwrapping process and decreases the DEM accuracy. Although the spatial and Doppler centroid decorrelation could be

\footnotetext{
${ }^{*}$ Corresponding author.

E-mail addresses: wangyang12nudt@163.com (Y. Wang), haifeng0728@vip.sina.com (H. F. Huang), dongzhen@vip.sina.com (Z. Dong), wumanqing@ustc.edu.cn (M. Q. Wu).
} 
reduced by applying space-varying minimum mean square error (SV-MMSE) filter [3] to SAR image pairs, a noise suppression filtering step applied to the interferometric phase is usually used before or together with the phase unwrapping step.

Generally speaking, interferometric phase filtering methods can be classified into three groups. The first group uses direct filtering without transformation and includes the methods in [4-10]. The second group transforms the interferometric phase into other domains and conducts the filtering [11-19]. The third group uses some comprehensive methods to simultaneously filter and unwrap the interferometric phase [20-24]. A classic method of the first group, the Lee method [4], is an anisotropic filter, which eliminates noise using local statistics and adaptive windows. Performance improvement is achieved by excluding singular pixels within a selected direction via the minimum mean squared error estimator in [5]. One method, which formulates the filtering process as a Bayesian estimation problem to utilize theoretical results and practical instruments, is developed in the statistical optimization framework of [6]. Vasile et al. invented an intensity-driven adaptive-neighbourhood method for denoising interferometric phase images in [7]. This method originally applied the region-growth method to intensity images in order to define the adaptive neighbourhood of a pixel to be filtered. An adaptive-contoured-window method is proposed in [8], which uses a low-pass filter along the local fringe orientation with an adaptive window. Because fringe and noise frequency distributions are different on the fringe contour, noise can be easily removed without disturbing the fringe signal. Interferometric phase filtering through a maximum a posteriori estimator that makes use of Gaussian Markov Random Fields (MRF) is proposed in [9]. Different from the classical permanent scatterers (PS) based techniques, this method is able to evaluate the height and deformation for any pixel of the scene. Interferometric phase noise suppression by incorporating InSAR magnitude information through 
a fractal surface scattering model is present in [10]. Spikes in areas with dense residues and long cliff-like artifacts for isolated residues are well corrected in the regularized DEM produced from the filtered phase.

The first frequency domain method is proposed in [11], which suppresses noise by multiplying the Fourier spectrum of a small phase patch by its smoothed absolute value to the power of a parameter. Although effective in flat regions, a lack of adaptation hinders its application in areas with low coherence. Baran et al. [12] addressed this problem by introducing coherence magnitude as the filtering parameter. Therefore, different areas are adaptively filtered according to their coherence. By exploring respective advantages, Wang et al. proposed a joint method using the modified Goldstein and simplified Lee method in [13]. This method is efficient and effective, particularly for interferometric phases with tightly packed fringes or low coherence. An adaptive multiresolution technique, based on the coherent summation principle, is proposed in [14]. By altering the size and shape of the interferogram resolution for frequency estimation and invalid estimation elimination, the fringes are properly filtered. The waveletdomain method is promising because the phase information and noise can be more easily separated in the wavelet domain. López-Martínez and Fàbregas proposed the first waveletdomain (WInPF) filter in [15], together with the complex phase noise model, which was of great importance to subsequent work. Based on this model, Zha et al. [16] proposed a wavelet-packettransform-based Wiener filter. Because phase information and noise can be further separated in the wavelet packet domain, this filter is superior to the WInPF filter. In fact, image filtering is an estimation problem, which is why Bian and Mercer [17] proposed two wavelet-domain methods using simultaneous detection and estimation. In order to further reduce the remaining impulse noise in the filtered phase obtained using the "Filtrage par Approche Multiéchelle Modifié" 
(FAMM) algorithm, an improved algorithm (EFAMM) is proposed in [18] in which more accurate noise mask values with respect to the InSAR coherence information are given. As the scenes in homogeneous areas exhibit a locally spatial stationarity which provides a rational of sparse representation of amplitude and interferometric phase to perform noise reduction, a joint denoising method using simultaneous regularization in the wavelet domain is proposed in [19]. Through a joint sparse constraint and iterations, the multi-channel data are refined, with phase discontinuities well preserved.

Based on a number of approximations and assumptions, the aforementioned two groups will inevitably add estimation error to the phase unwrapping step. To avoid this problem, some methods treat denoising and unwrapping as a single true phase recovery problem. A fifth complex-valued MRF model and residue-based adaptive mask, which is used to reduce the number of residues, are used in [20]. A method based on Kalman filtering is proposed in [21]. Instead of an interferometric phase, a complex interferogram is used in combination with the phase slope information obtained from the power spectral density. As a result, this method implicitly realizes directional phase filtering without removing geometrically induced residues in under-sampled sloped regions. By incorporating a nonlinear and non-Gaussian noise model, Martinez-Espla et al. propose a particle filter in [22]. Their results exhibited a significant improvement with respect to other conventional unwrapping methods in certain situations. The phase local approximation (PhaseLa) technique [23] applies a local polynomial approximation to $\cos (\square)$ and $\sin (\square)$ arguments to estimate the absolute phase from noisy wrapped phase data. In addition, it utilizes the intersection-of-confidence-interval method to adaptively select pointwise varying window size. Taking a priori knowledge of interferometric phase into account, Chen et al. [24] used MRF to model the relationship between the elements in a random variable set, 
including both the true phase and its observations. After the model is built, a new energy function is defined according to the local-independence property inferred from the MRF structure. It is then minimized to obtain an estimate of the true phase value.

Taking the interferometric stacks into consideration, some first papers have been published about the adaptive filtering of those stacks recently [25-29]. Ferretti et al. [25] proposed DespecKS, a space adaptive processing embedded in their SqueeSAR procedure that works on amplitude SAR data and identifies statistically homogeneous areas in which the interferometric phase could be filtered properly. Different methods of selecting statistically similar pixels based on their amplitude distribution for the purpose of adaptive multi-looking have been compared in [26] and recommendations are made concerning which method to use in practice. An adaptive multi-looking method for airborne single-pass multi-baseline InSAR stacks is proposed in [27]. This method determines the homogeneous areas through a principal component analysis-based thresholding strategy which is both fast and efficient for the estimation of complex covariance matrices of InSAR data stacks. One method to produce robust denoised wrapped phase time series from the combination of wrapped interferograms is proposed in [28]. Instead of coherence, a new quality map, colinearity, is defined and used to obtain the weighted average wrapped phase, multi-link SAR (MuLSAR) interferogram, and the high-frequency patterns are so well preserved that this technique holds promise to help retrieve maximum information out of the large database. A simple phase filtering approach that can effectively increase the number of pixel with a temporal coherence value greater than 0.7 is proposed in [29] to improve the performance of classical extended minimum cost flow-small baseline subset (EMCF-SBAS) inversion technique. In addition, this approach is parallelizable, thus allowing a significant reduction of the overall algorithm running time. 
Although the aforementioned methods are excellent in some aspects, a better filtering performance can be achieved via exploiting more information from the InSAR data. In general, images contain repetitive structures, such as corners and lines. By exploring those redundancies in an image, the filtering performance can be improved. In 2005, Buades et al. [30] proposed a new method, the non-local means (NLM), for image filtering. The key to this method is that the denoised value at $x$ is a mean of the values of all points whose Gaussian neighbourhood looks like the neighbourhood of $x$. Since its introduction, NLM has been modified for many applications [31-36]. The first InSAR application was the non-local interferometric SAR (NLInSAR) estimator [37]. This method uses a non-local approach for the joint estimation of the reflectivity, interferometric phase and coherence maps from an interferometric pair of coregistered single-look complex (SLC) SAR images. Adopting the non-local paradigm, NLInSAR introduces a patch similarity criterion, which is suitable for SLC images, and a weighted maximum likelihood estimation of the SAR interferogram, with weights derived in a data-driven manner. By applying NLM to TanDEM-X data, Zhu et al. [38], [39] obtained encouraging results, in which improved DEMs possessed a quality similar to the HRTI-4 (high-resolution terrain information) standard. Recently, the NL-InSAR has been extended to a more general framework, non-local SAR (NL-SAR) [40], that handles amplitude (SAR), interferometric (InSAR), polarimetric (PolSAR) or polarimetric and interferometric (PolInSAR) images in a unified way. The NL-SAR is based on the building of extended non-local neighbourhoods that are defined on the basis of pixel similarity as evaluated by multi-channel comparison of image patches. Through a bias reduction step and an unsupervised adaptation strategy, this method is able to obtain the best non-local estimation to form a single restored image with good preservation of radar structures and discontinuities. Furthermore, this method could be adapted to 
very high resolution images in which the noise is known to depart from Goodman's model. Other InSAR applications can be found in [41-43].

Using both geometrically and photometrically similar patches, Chatterjee and Milanfar [44] extended the NLM and proposed the patch-based locally optimal Wiener (PLOW) method. By utilizing patch-based calculations and residual filtering, PLOW is expected to be on par or exceed the NLM. In this paper, we propose a new method for interferometric phase filtering. Accounting for the characteristics of the phase map, we modify PLOW to better suit the interferometric phase. We use the coherence map for adaptive clustering and cluster mean estimation, and utilize an adaptive mean estimator to calculate the local noise covariance. This paper is organized as follows. Section 2 reviews the interferometric phase model in real and complex domains, respectively. Section 3 gives a brief introduction to the motivation behind and implementation of PLOW. Modifications are described in Section 4. Section 5 compares the results of the proposed method with the PLOW and NL-InSAR methods for both simulated and real data. Future work is discussed in Section 6. Section 7 presents our conclusions.

\section{Phase model}

As validated by Lee [4], an interferometric phase in the real domain can be characterized by an additive noise mode as

$$
\theta_{z}=\theta_{x}+v
$$

where $\theta_{z}$ is the noisy phase, $\theta_{x}$ is the real phase without noise, $v$ is the noise with a zero mean and standard deviation $\sigma_{v}, \theta_{x}$ and $v$ are independent of each other. Based on the InSAR phase measuring mechanism, the interferometric phase occurs in the interval of $[-\pi, \pi)$. Therefore, direct filtering in the real domain cannot be applied because of phase jumps. Phase jumps, where 
the phase value goes from $-\pi$ to $\pi$ or $\pi$ to $-\pi$, represent high-frequency information and should be preserved in order to correctly reconstruct the absolute phase. To solve this problem, the interferometric phase should be processed in other domains where the phase jumps can be preserved.

According to the detailed analysis presented in [15], the interferometric phase in the complex domain is continuous, and the phase noise model in this domain can be written as

$$
\begin{aligned}
& \operatorname{Real}_{z}=\cos \left(\theta_{z}\right)=N_{c} \cos \left(\theta_{x}\right)+v_{r} \\
& \operatorname{Imag}_{z}=\sin \left(\theta_{z}\right)=N_{c} \sin \left(\theta_{x}\right)+v_{i}
\end{aligned},
$$

where $\operatorname{Real}_{z}$ and $\operatorname{Imag}_{z}$ are real and imaginary parts of $\theta_{z}$, respectively, while $v_{r}$ and $v_{i}$ can be considered as zero-mean additive noise and are independent from $\theta_{x} . N_{c}$ is the interferometric phase quality indicator, which monotonically increases with the coherence magnitude. Therefore, both the real and imaginary parts are filtered, and the final filtered phase can be extracted.

\section{Principle of PLOW}

PLOW is a patch-based method and proposed in [44]. Similar to the pixel-based method, the additive noise observation model of the patch-based method can be written as [44]

$$
\mathbf{y}_{i}=\mathbf{z}_{i}+\boldsymbol{\eta}_{i}
$$

where $\mathbf{y}_{i}$ and $\mathbf{z}_{i}$ are the noisy and noise-free image patches centred at $i$, and $\boldsymbol{\eta}_{i}$ is the noise patch. Accounting for white Gaussian noise, the linear minimum mean square error (LMMSE) estimator of each patch, $\mathbf{z}_{i}$, from its noisy observation, $\mathbf{y}_{i}$, can be written as [44]

$$
\hat{\mathbf{z}}_{i}=\overline{\mathbf{z}}+\mathbf{C}_{\mathbf{z}} \mathbf{C}_{\mathbf{y}}^{-1}\left(\mathbf{y}_{i}-\overline{\mathbf{z}}\right)
$$

where $\overline{\mathbf{z}}$ and $\mathbf{C}_{\mathbf{z}}$ are the mean and covariance of $\mathbf{z}_{i}$, and $\mathbf{C}_{\mathbf{y}}$ is the covariance of $\mathbf{y}_{i}$. 
Natural images exhibit repetitive patterns, such as geometric and photometric similarities, which can used to improve the filtering performance of Eq. (4). The geometrically similar patches have similar geometric structures and the photometrically similar patches are multiple observations of a single latent patch with the differences arsing (ideally) due to noise only (photometric similarity is a stricter condition than geometric similarity) [44]. These similarities are obvious in the interferometric phase and an example is shown in Fig. 1(a), in which 3

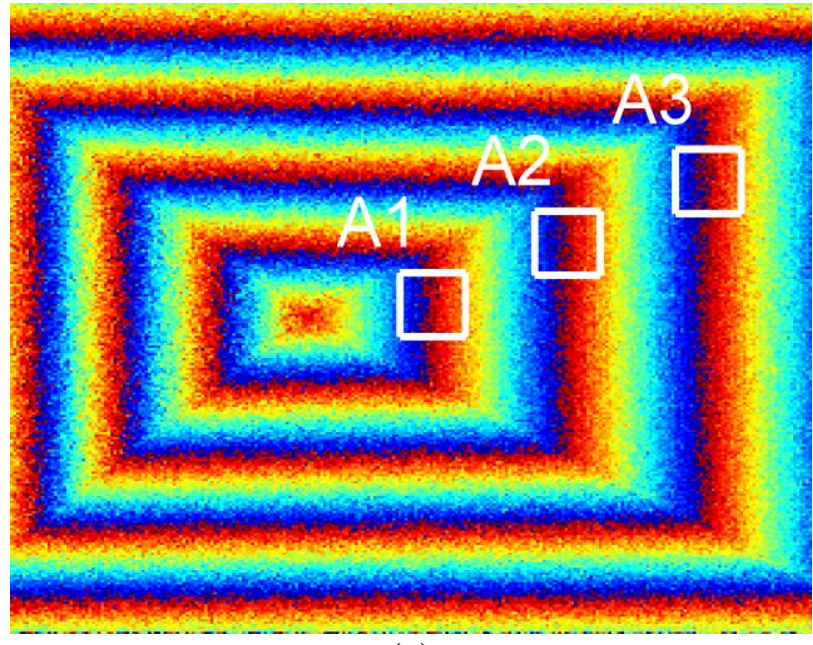

(a)
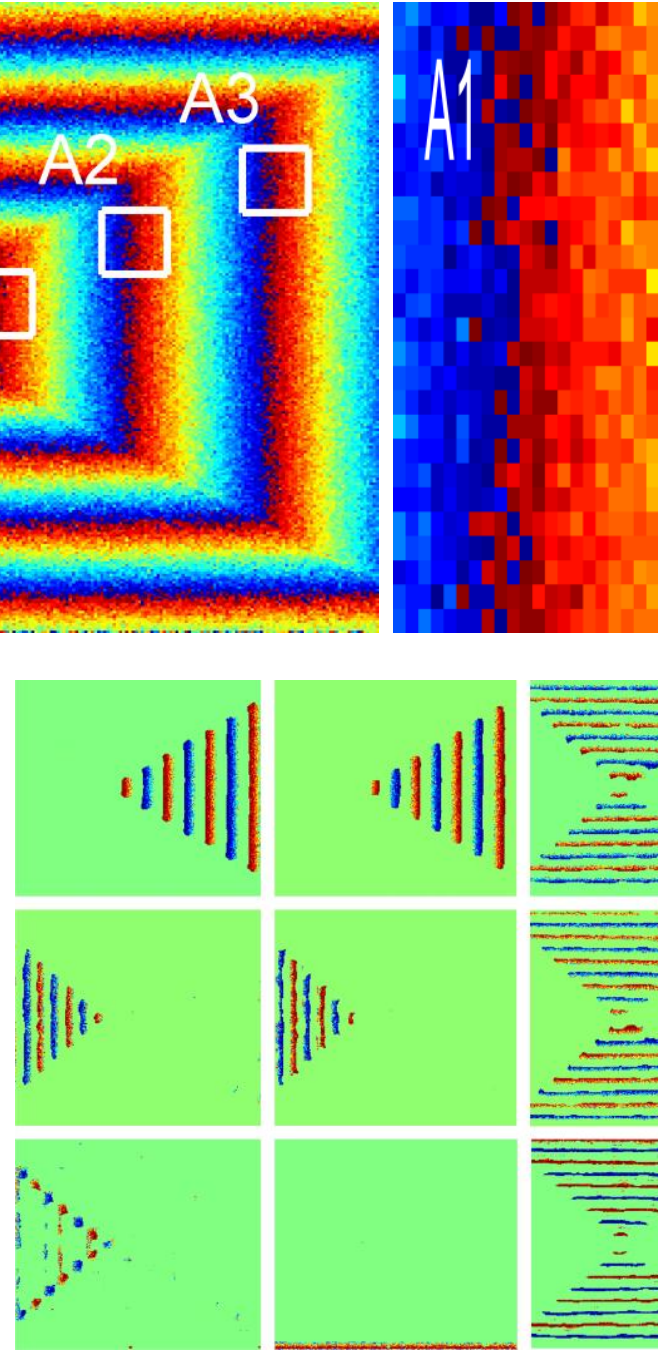

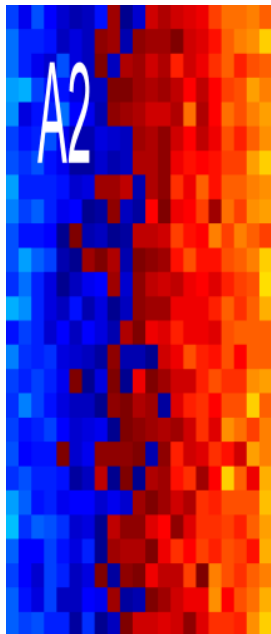

(b)

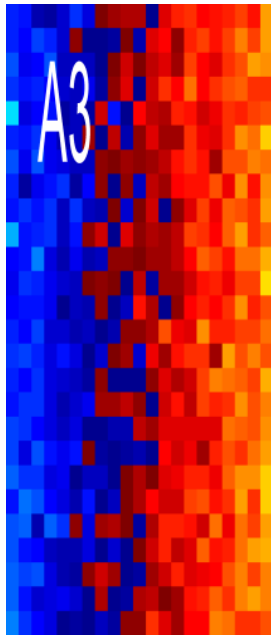

Fig. 1. Illustration of photometric and geometric similarities. (a) Simulated interferometric phase with 3 photometrically similar patches labelled out. (b) Enlargement of photometrically similar patches labelled in (a). (c) Clustering results of (a) based on geometric similarity, with cluster number $\mathrm{K}=9$. Pixels in any particular cluster can have quite different intensities but similar geometric structure. 
photometrically similar patches marked 'A1', 'A2', and 'A3' are labelled out. For clarity, the 3 patches are enlarged in Fig. 1(b). Fig. 1(c) illustrates the clustering results of each pixel based on its geometric similarity. The clustering process is as follows: 1) the locally adaptive regression kernels (LARK) [45], [46] feature of each pixel is calculated from the image patch $(11 \times 11$ pixels) centered at that pixel; 2) using the LARK features, we run K-means [47] to cluster the interferometric phase. As the LARK features are robust to differences in contrast and intensity, any two pixels within one cluster of Fig. 1(c) can have quite different intensities but similar geometric structure.

By using these similarities and mathematical processing, the PLOW estimate, derived from Eq. (4), can be written as

$$
\hat{\mathbf{z}}_{i}=\overline{\mathbf{z}}+\left[\mathbf{I}-\left(\sum_{j} \omega_{i j} \mathbf{C}_{\mathbf{z}}+\mathbf{I}\right)^{-1}\right] \sum_{j} \frac{\omega_{i j}}{\sum_{j} \omega_{i j}}\left(\mathbf{y}_{j}-\overline{\mathbf{z}}\right),
$$

where $\mathbf{I}$ is the identity matrix, $\overline{\mathbf{z}}$ is the cluster mean estimated using all patches within one cluster and $\omega_{i j}$ is the denoising weight, which determines the contributing factor for patch $\mathbf{y}_{j}$ in denoising the reference patch, $\mathbf{y}_{i}$, with the most similar patches exerting the greatest influence. $\mathbf{C}_{\mathbf{z}}$ is estimated from the noisy patches within one cluster as [44]

$$
\hat{\mathbf{C}}_{\mathbf{z}}=\left[\hat{\mathbf{C}}_{\mathbf{y}}-\sigma^{2} \mathbf{I}\right]_{+},
$$

where $[\mathbf{M}]_{+}$denotes matrix $\mathbf{M}$ with its negative values replaced by zero or a very small positive value, $\hat{\mathbf{C}}_{\mathbf{y}}$ is estimated using a simple estimator [44], and $\sigma^{2}$ is the noise covariance which is estimated using a gradient-based median estimator [44]. When all the patches have been filtered using Eq. (5), the estimates from overlapping image patches are integrated to achieve the final results for each pixel using a weighted least-square framework. The whole filtering process of 
PLOW consists of geometric clustering, cluster moments estimation, weights calculation and estimation aggregation and readers may refer to [44] for detailed information.

4 Modified PLOW for the interferometric phase

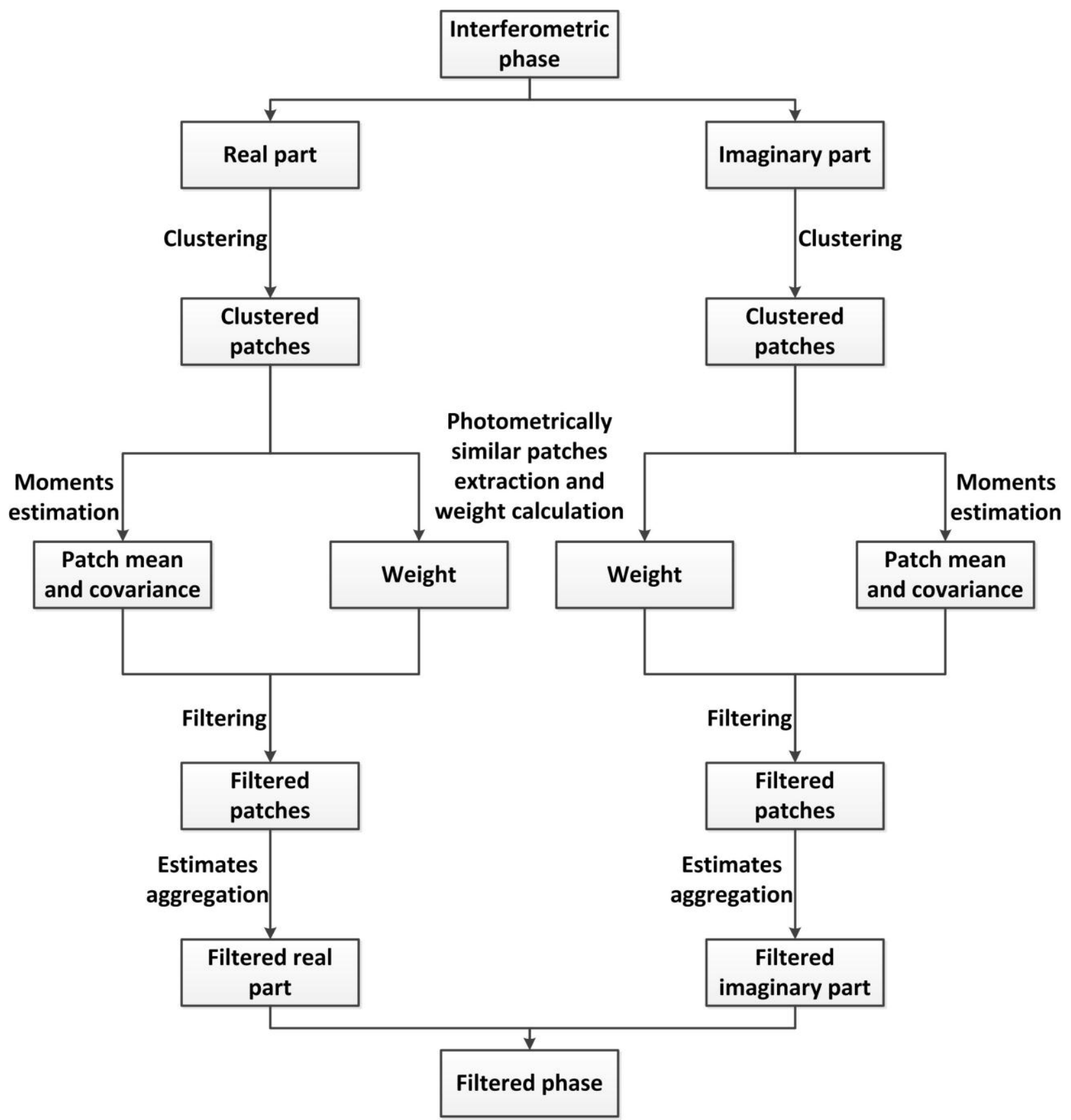

Fig. 2. Flowchart of the proposed method. 
The flowchart of the proposed method is shown in Fig. 2. Compared with the PLOW method, three modifications are proposed and elaborated as follows.

\subsection{Coherence-based clustering}

As described in [44], pixels are clustered using K-means according to their LARK features. As noted in [46], the value of K, namely the number of clusters (NOC), chosen affects the denoising results. In summary, too few clusters could result in the incorrect estimation of the moments because structurally dissimilar patches are clustered together; while too many clusters lead to too few patches within each cluster, making the moment estimation less robust [44]. Taking this consideration and through extensive experiments, a fixed value of $\mathrm{K}=15$ is determined and used in [44]. This value yields good results for many cases, with the mean squared error (MSE) fairly close to that obtained by tuning the NOC for each particular case [44]. Although LARK features are resistant to noise, as well as differences in contrast and intensity, clustering errors due to strong noise cannot be fully avoided [44]. Then, the use of K-means also has intrinsic drawbacks. First, when there is an insufficient amount of data to be clustered, initial clustering will affect the final clustering results significantly. Second, although iterative partitioning attempts to minimize the sum of the within-cluster sums of point-to-cluster-centroid Euclidean distances, finding a global minimum is not guaranteed. Third, K-means is sensitive to outliers and noise. Therefore, data located far from the centroid may shift the centroid away from the real value, thereby influencing the results.

As for the interferometric phase, the coherence map, which is estimated from two coregistered SAR images, is an indicator of phase quality. For a single-look interferometric phase, the variance of phase noise, $\sigma_{\theta}^{2}$, is related to the coherence magnitude, $|\rho|$, through [1] 


$$
\sigma_{\theta}^{2}=\frac{\pi^{2}}{3}-\pi \arcsin (|\rho|)+\arcsin ^{2}(|\rho|)-\frac{L i_{2}\left(|\rho|^{2}\right)}{2}
$$

where $L i_{2}(\square)$ is Euler's dilogarithm. Therefore, using the estimated coherence for interferometric phase filtering is well-reasoned and has been utilized in many studies, including [4], [5], [12], [15] and [24]. In this study, we propose a coherence-based clustering method. We first estimate the coherence map using the maximum likelihood estimator [1] as

$$
|\rho|=\frac{\left|\sum_{m=1}^{M} \sum_{n=1}^{N} I_{1}(m, n) I_{2}(m, n)\right|}{\sqrt{\sum_{m=1}^{M} \sum_{n=1}^{N}\left|I_{1}(m, n)\right|^{2} \sum_{m=1}^{M} \sum_{n=1}^{N}\left|I_{2}(m, n)\right|^{2}}},
$$

where $I_{1}$ and $I_{2}$ are two coregistered SAR images and $M=N=5$. Then, we obtain the minimum and maximum values of that map. Finally, the NOC is determined by

$$
\mathrm{NOC}=\operatorname{int}\left|\frac{c o h_{\max }-c o h_{\min }}{a_{\text {interval }}}\right| \text {, }
$$

where int $/ \mid$ rounds toward positive infinity, $c o h_{\max }$ and $c o h_{\min }$ are the maximum and minimum coherence magnitude, respectively, and $a_{\text {interval }}$ is set to 0.1 and used in the remainder of this paper. Finally, each pixel is clustered according to its coherence magnitude. Additionally, we note that if there are too few pixels in one cluster, then the cluster can be merged with neighbouring clusters, or the entire image can be re-clustered using a larger interval such, as 0.15 . Using the coherence for clustering has at least four advantages. First, the performance of the LARK features deteriorates as noise increases, but this is not the case for coherence. According to Eq. (7), we utilize the noise level (variance) for clustering, rather than using features that are resistant to noise. Second, because the NOC is mainly determined by the coherence distribution of the phase, the coherence-based clustering is adaptive. Third, less time is needed using 
coherence-based clustering. When $a_{\text {interval }}=0.1$, the NOC of a phase can be no greater than 10 because the coherence magnitudes lie in the interval of $[0,1]$. Finally, because noise is spatially variant in the interferometric phase, patches within one cluster obtained using PLOW may have significantly different noise levels, which makes moments estimation inaccurate. On the contrary, moments can be more accurately estimated using patches of similar coherence magnitude values (noise levels). This gives rise to the second modification, which will be discussed in the next section.

\subsection{Locally adaptive mean estimator for noise covariance}

To obtain $\hat{\mathbf{C}}_{\mathbf{z}}$ from Eq. (6), $\sigma$ needs to be estimated using a gradient-based median estimator according to [44]

$$
\hat{\sigma}_{\text {median }}=1.4826 \text { median }(|\nabla \mathbf{Y}-\operatorname{median}(\nabla \mathbf{Y})|)
$$

where ' median $(\mathbf{M})$ ' is to obtain the median value of $\mathbf{M} ; \nabla \mathbf{Y}$ is the vectorized form of the gradient of the entire image $\mathbf{Y}$, which is equivalent to say that this estimator is a global one. Moreover, this estimator is based on the median absolute deviation [48] and developed under the assumption that the noise is spatially invariant through the entire image, which is not the case for interferometric phase noise. Therefore, this parameter should be estimated locally and adjusted to obtain a better filtering performance. This modification should be applied according to some factors, such as the level of spatial coherence variability and fringe densities of the phase data. Taking the scene-wide magnitude of noise into consideration, Bian and Mercer [17] multiply $\hat{\sigma}_{\text {median }}$ by a prespecified scaling parameter $\lambda$ which mostly takes values between 1 and 2 . In essence, Bian is trying to suppress spatially variant noise with an overestimation of Eq. (10) but does not give instructions on how to choose the value of $\lambda$. The best result for a specific case 
may be obtained by manually tuning the value of $\lambda$ which is not adaptive. Here, we propose a locally adaptive mean estimator for noise covariance. Specifically, we use a local estimator that calculates noise covariance of each cluster using all image patches within that cluster as

$$
\hat{\sigma}_{\text {mean }}=1.4826 \text { mean }\left(\left|\nabla \mathbf{Y}_{k}-\operatorname{mean}\left(\nabla \mathbf{Y}_{k}\right)\right|\right)
$$

where ' mean $(\mathbf{M})$ ' is to obtain the mean value of $\mathbf{M}$ and $\mathbf{Y}_{k}$ includes all the image patches in the $k$ th cluster.

\subsection{Coherence-weighted mean of cluster}

After clustering, mean of underlying noise-free cluster can be approximated by the expectation of the noisy patches within each cluster as [44]

$$
\hat{\overline{\mathbf{z}}}=E\left[\mathbf{y}_{i} \in \mathbf{\Omega}_{k}\right] \approx \frac{1}{M_{k}} \sum_{\mathbf{y}_{i} \in \mathbf{\Omega}_{k}} \mathbf{y}_{i}
$$

where $\boldsymbol{\Omega}_{k}$ denotes the $k$ th cluster with $M_{k}$ image patches. This is the arithmetic mean without considering the pixel quality. Obviously, pixels of higher quality should exert more influence on the result, while the influence of low quality pixels should be suppressed. Moreover, PLOW is derived under the assumption that noise is Gaussian and spatially invariant. However, interferometric phase noise is spatially variant and could be non-Gaussian. Although the presence of spatially variant and non-Gaussian noise degrades the performance of the arithmetic mean, a weighted mean is much more robust. Therefore, we use the coherence map as weights and modify Eq. (12) as

$$
\hat{\mathbf{z}}_{w}=\frac{1}{\sum_{i=1}^{M_{k}} \operatorname{coh}_{i}} \sum_{i=1}^{M_{k}} \operatorname{coh}_{i} \mathbf{y}_{i},
$$

where $\mathbf{c o h}_{i}$ is the coherence map of image patch $\mathbf{y}_{i}$. 


\section{Experimental results and analysis}

In this section, we conduct experiments using simulated and real data to test the effectiveness and robustness of the proposed method. The results obtained using the PLOW and NL-InSAR methods are also listed for comparison. In terms of evaluation, both visual inspection and quantitative indexes are used. The indexes include the MSE, SSIM (structural similarity) map [49] with its mean value MSSIM, number of residues (NOR) [50] and running time (T).

Because natural image signals are highly structured, their pixels exhibit strong dependencies, especially when they are spatially proximate. These dependencies carry important information regarding the structure of the objects in the visual scene [49]. Therefore, based on pointwise signal differences, which are independent of the underlying signal structure, MSE is not well matched to perceived visual quality. Unlike MSE, SSIM takes advantage of known characteristics of the human visual system and considers image degradations as perceived changes in structural information variations, so that errors are penalized in accordance with their visibility. In practice, one typically requires a single overall quality measure for the entire image; therefore an MSSIM value is also calculated.

All of the experiments were performed using a PC with an Intel Core2 Quad CPU 3.0-GHz with 8-GB memory. The publicly available codes of the PLOW (http://users.soe.ucsc.edu/ priyam/PLOW/) and NL-InSAR (http://www.math.ubordeaux1.fr/ cdeledal/nlinsar.php) methods are directly used, with parameters set to their default values. The main parameters of the PLOW method are as follows: a search window size of $30 \times 30$ pixels, a patch size of $11 \times 11$ pixels, and a NOC of 15 . The main parameters of the NL-InSAR method are as follows: a search window size of $21 \times 21$ pixels, a patch size of $7 \times 7$ pixels, a minimum equivalent number of looks of 10 , and a number of iterations of 10 . The main 
parameters of the proposed method are almost the same as those of the PLOW method except for the NOC. A simple speedup that denoises very third patch [44] is applied to the PLOW and proposed methods.

\subsection{Simulated data}

To examine the filtering performance of the proposed method, one simulated data set of $512 \times 512$ pixels was generated using the InSAR MATLAB toolbox [51]. The DEM for a rugged

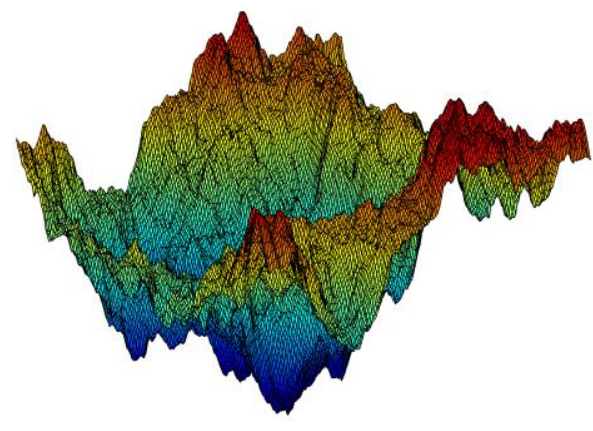

(a)

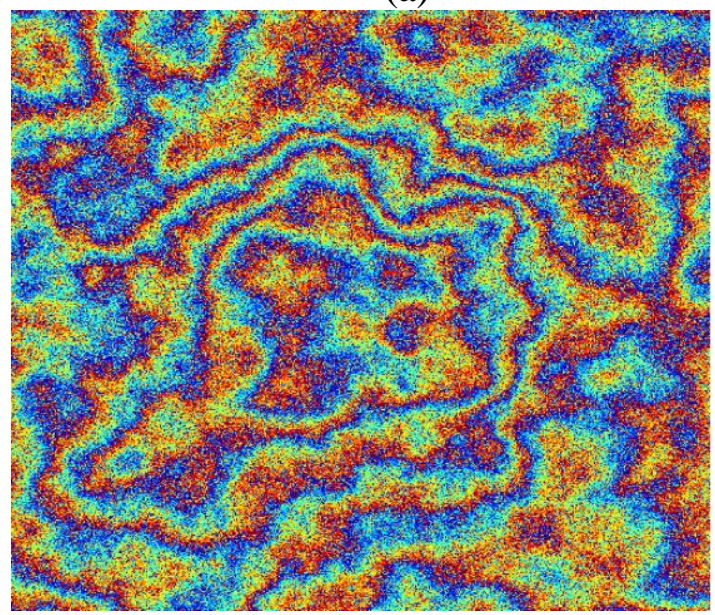

(c)
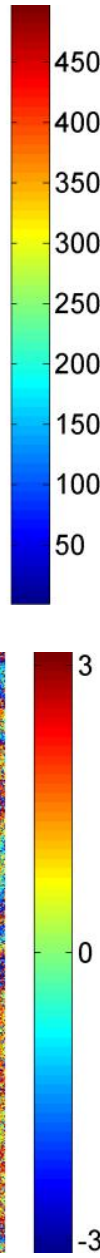

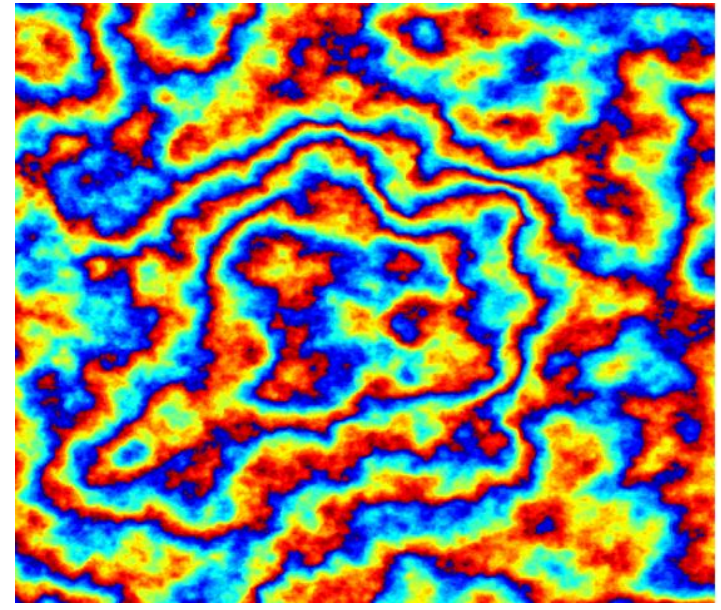

(b)

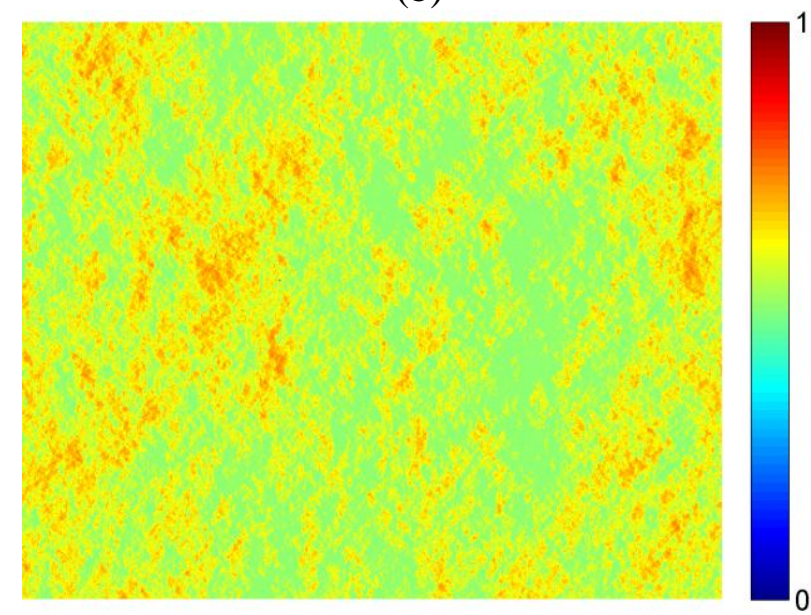

(d)

Fig. 3. Simulated data. (a) DEM. (b) Noise-free phase. (c) Interferometric phase. (d) Coherence map.

terrain is shown in Fig. 3(a), and the corresponding noise-free phase is shown in Fig. 3(b). With noise added, the interferometric phase is shown in Fig. 3(c), with its corresponding coherence 
map shown in Fig. 3(d). The NOR of Fig. 3(c) is 45142, and the mean value of the coherence map is 0.5366 , which is common for spaceborne InSAR data.

Filtered phases obtained using the PLOW, NL-InSAR and the proposed methods are shown in Figs. 4(a)-(c), respectively. From the coarse view of these figures, we can observe that noise is

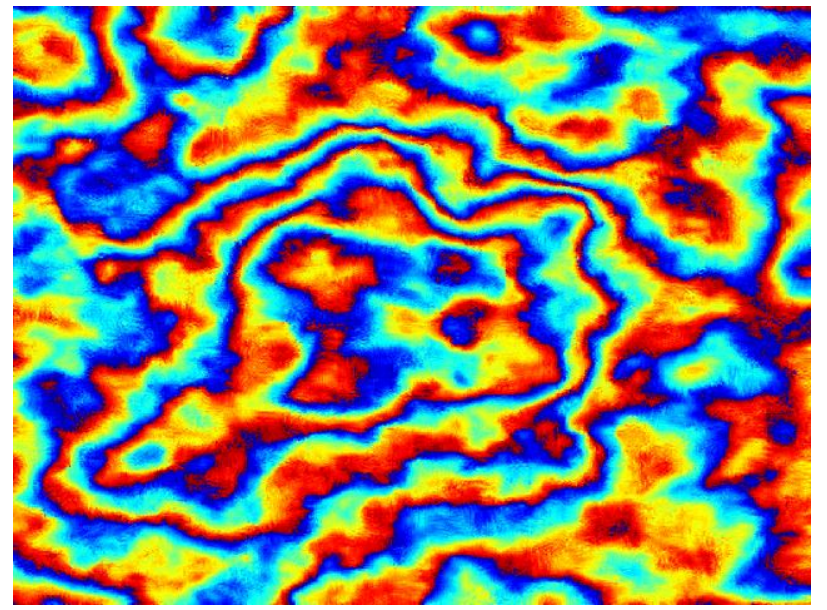

(a)

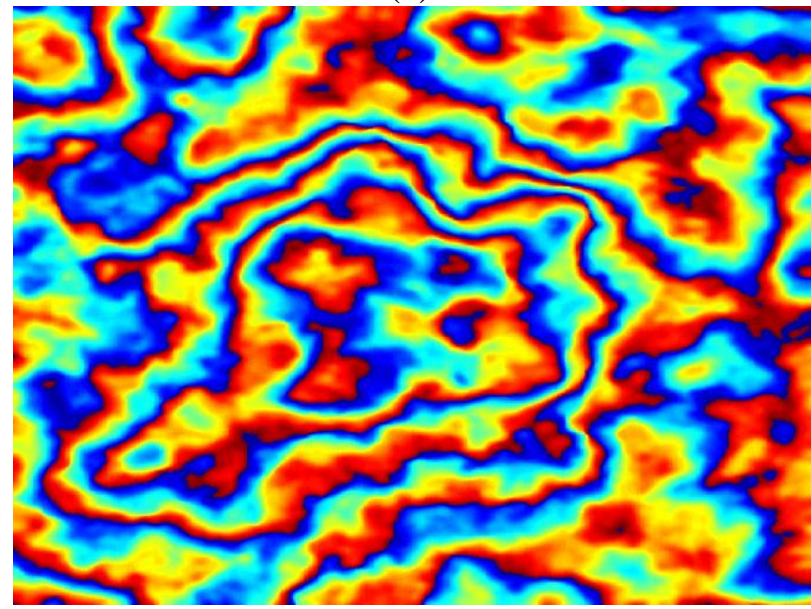

(c)

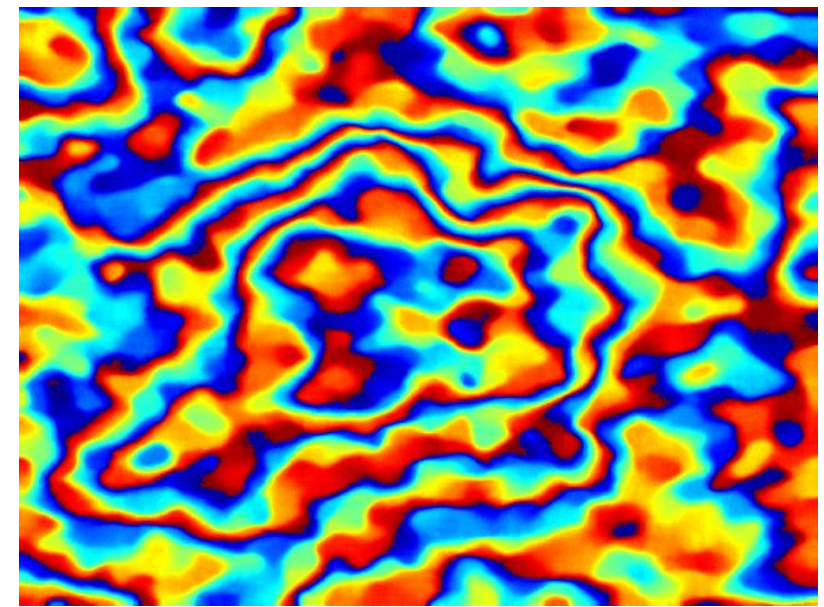

(b)

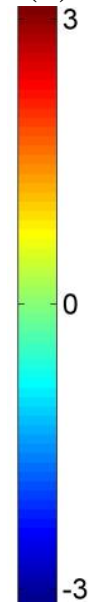

Fig. 4. Filtered phase obtained using the (a) PLOW, (b) NL-InSAR and (c) proposed methods. well suppressed in all filtered phases. Note that Fig. 4(a) is slightly under-filtered and Fig. 4(b) is slightly over-filtered while Fig. 4(c) reaches a balance between Figs. 4(a) and (b). To demonstrate this inference and more clearly illustrate the difference, phase differences (noisefree phase minus filtered phase) are present in Fig. 5, in addition to the fitted histogram curves of each phase difference. Fig. 5 suggests that the PLOW and NL-InSAR methods exhibit 
comparable phase difference results. However, the proposed method is better than the PLOW and NL-InSAR methods because the fitted curve of the proposed method is sharper and more centred on zero than those of the two competing methods.

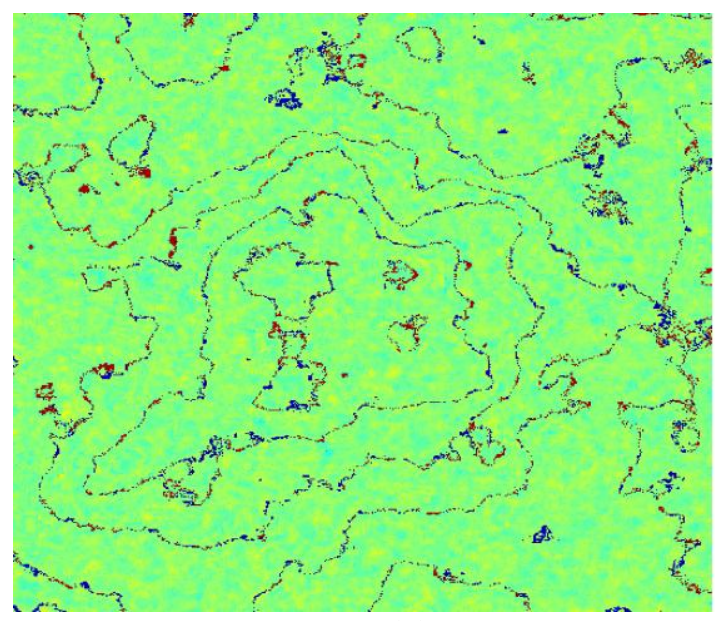

(a)

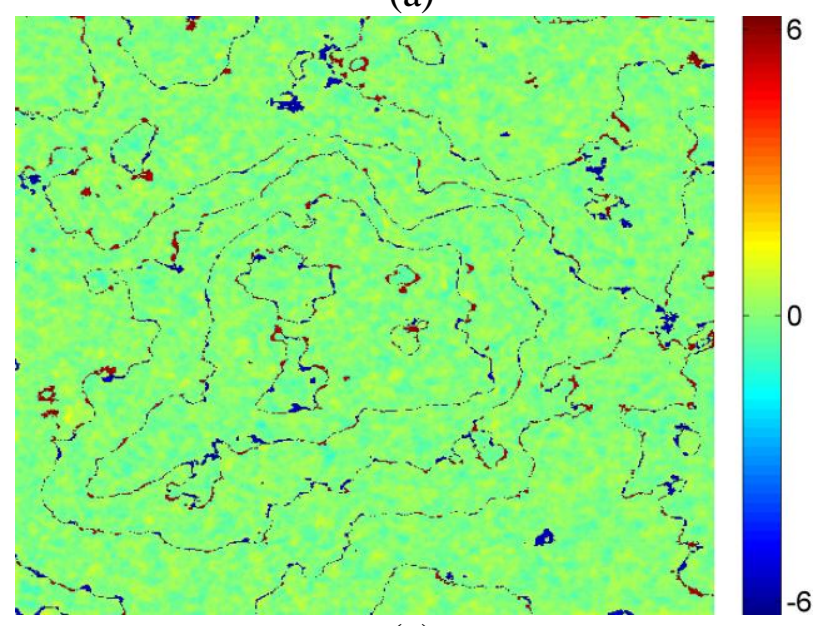

(c)

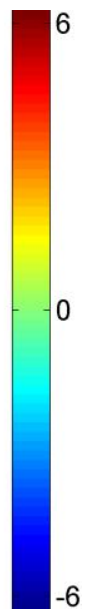

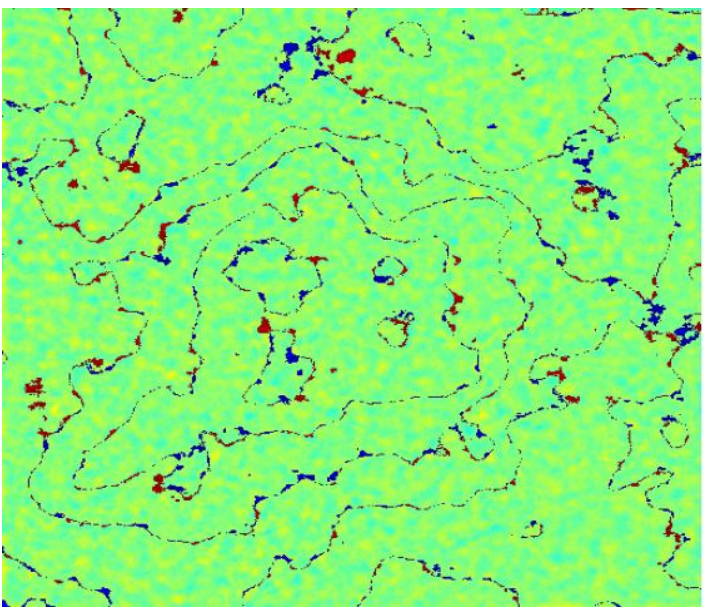

(b)

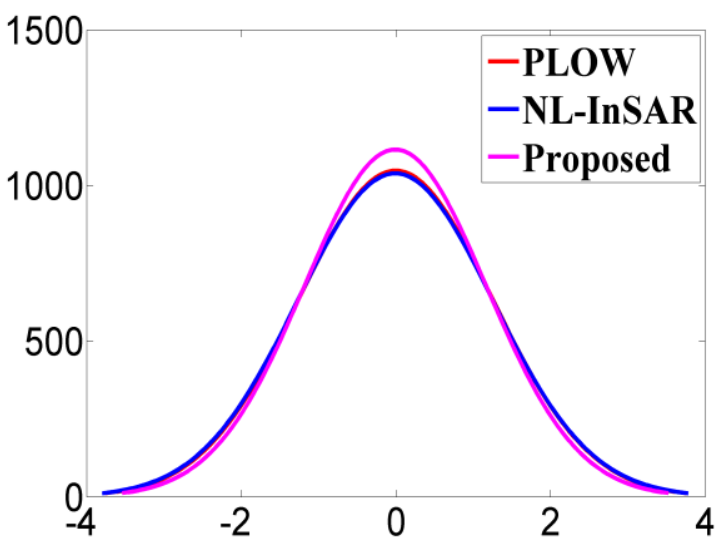

(d)

Fig. 5. Phase difference and fitted histogram obtained using each method. (a) Phase difference of PLOW method. (b) Phase difference of NL-InSAR method. (c) Phase difference of proposed method. (d) Fitted histogram curves of (a), (b) and (c).

To analyse details preservation, an SSIM map is calculated and shown in Fig. 6. This index is distributed in the interval of $[-1,1]$, with 1 representing the best quality and -1 representing the worst. Via comparing Figs. 6(b) and (d), one can see that differences exist between the PLOW 


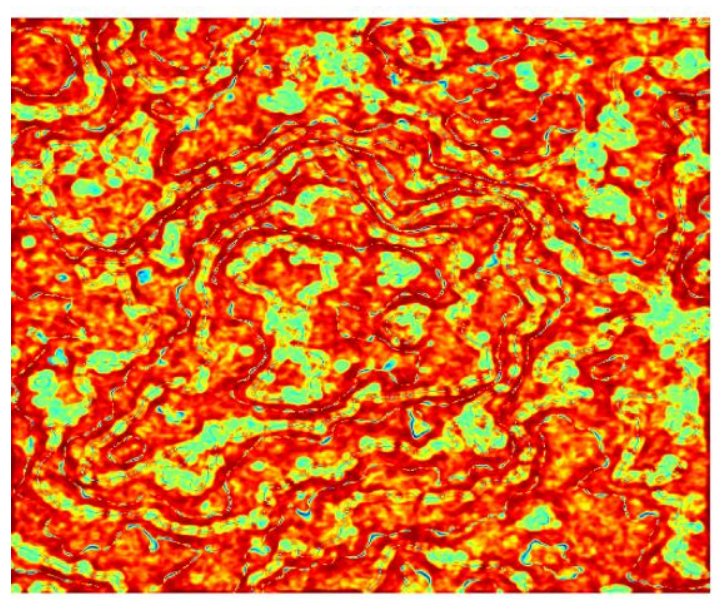

(a)

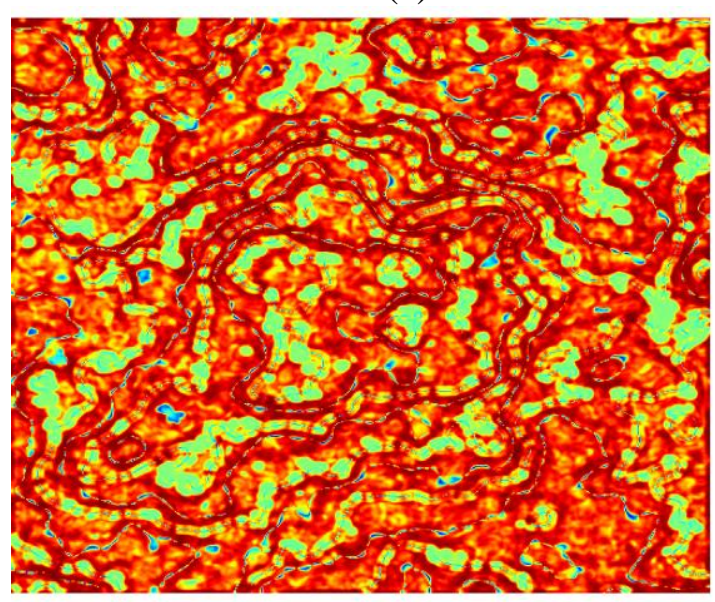

(c)

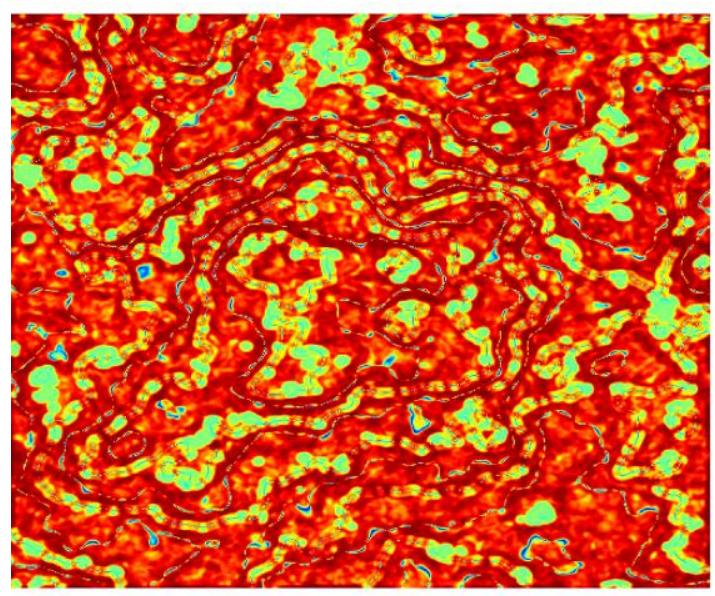

(e)

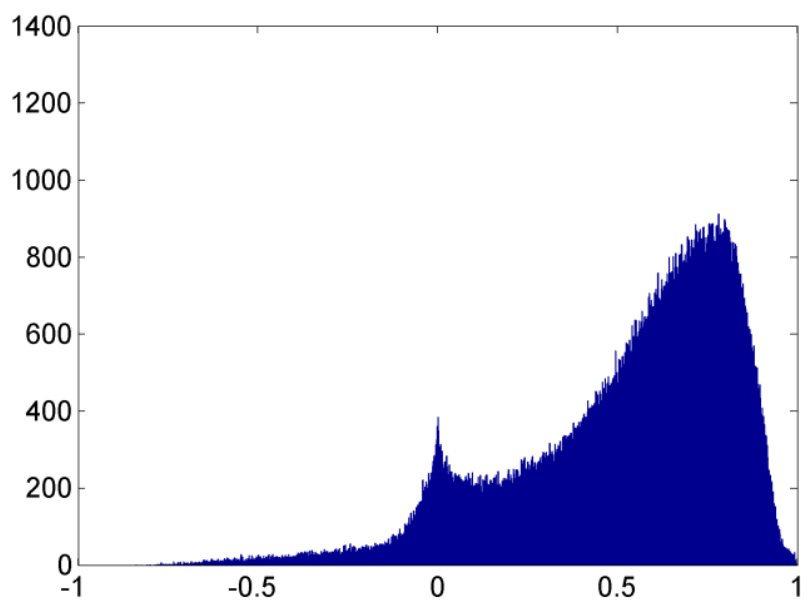

(b)

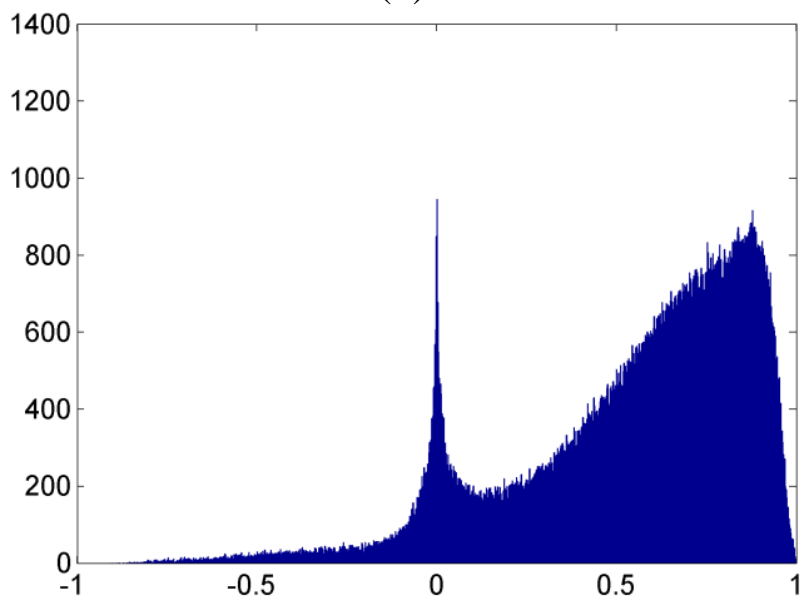

(d)

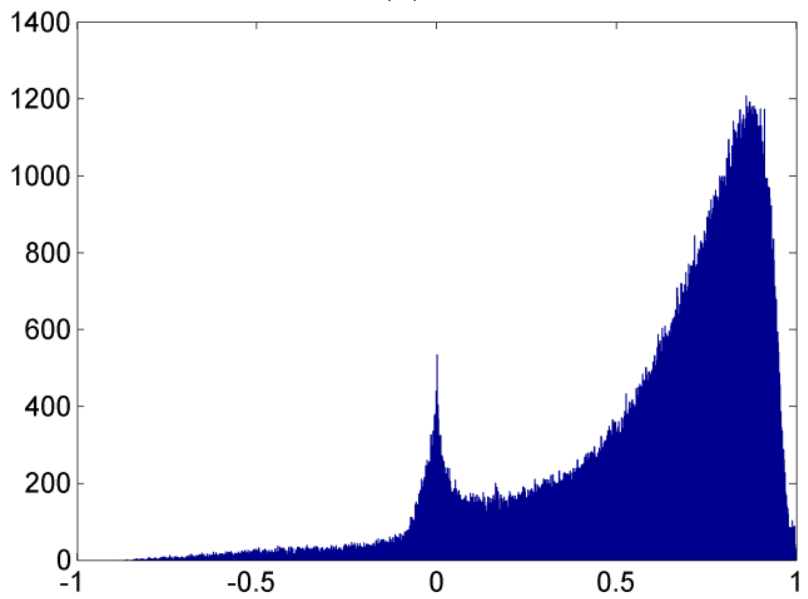

(f)

Fig. 6. SSIM map and its histogram obtained using each method. (a) SSIM map and (b) its histogram of PLOW method. (c) SSIM map and (d) its histogram of NL-InSAR method. (e) SSIM map and (f) its histogram of proposed method.

and NL-InSAR methods. NL-InSAR tends to have more pixels, with an SSIM coefficient distributed around 0 and in the interval of $[0.75,1]$. However, pixels from Fig. 6(a) are more 
centralized in the interval of $[0,0.75]$. Clearly, the proposed method exhibits a significant advantage over the PLOW and NL-InSAR methods because there are significantly more pixels in Fig. 6(f) than in Figs. 6(b) and (d), whose SSIM coefficient is distributed in the interval of $[0.6,1]$. As indicated by Wang [49], structural information is one of the most important pieces of information that an image filtering method should maintain. If the structural information is well preserved, then the original information could be nearly fully recovered via a simple process [49]. As in the interferometric phase, phase gradients or derivatives are types of structural information, which play key roles in gradient-based phase unwrapping methods [50]. In addition to the phase gradients, other structural information is both directly or indirectly used in various phase unwrapping methods. Therefore, the structural information plays an important role in phase unwrapping, and the filtering method should change the structural information as little as possible. From this point of view, the proposed method is superior to the other two methods.

The aforementioned evaluation indexes are applied for quantitative comparison. From the statistical results (Table 1), we can see that the proposed method obtains the lowest MSE and highest MSSIM. Therefore, the filtered phase obtained using the proposed method has the lowest bias and is closest to the noise-free phase. With respect to running time, we note that it is hard to make a fair comparison between the PLOW and NL-InSAR methods because the codes of those two methods are written in MATLAB/C/MEX. However, comparison between the PLOW and proposed methods could be made because the latter is derived from the former. As seen from Table 1, the proposed method needs less running time than the PLOW method because feature extraction is not needed and fewer clusters are obtained using the proposed method. Therefore, the total running time of the proposed method is reduced.

\section{Table 1}


Quantitative comparison between three methods on simulated data

\begin{tabular}{|c|c|c|c|c|}
\hline Method & MSE & MSSIM & NOR & T (s) \\
\hline No filtering & 1.2154 & 0.0534 & 45142 & - \\
\hline PLOW & 0.3252 & 0.5208 & 18 & 49 \\
\hline NL-InSAR & 0.3191 & 0.5429 & 0 & 410 \\
\hline Proposed & 0.2811 & 0.5963 & 0 & 22 \\
\hline
\end{tabular}

\subsection{Real data 1}

In this section, we used the interferometric phase of the SIR-C/X-SAR real data, with a size of $100 \times 250$ pixels $\left(X-b a n d\right.$, baseline length $=60 \mathrm{~m}$, incidence angle $\approx 49^{\circ}$, Etna Volcano, Italy), to confirm the effectiveness of the proposed method. The amplitude map of master image, interferometric phase and corresponding coherence map are shown in Figs. 7(a)-(c), respectively.

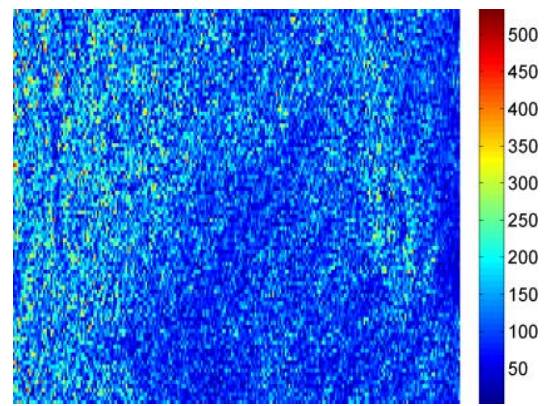

(a)

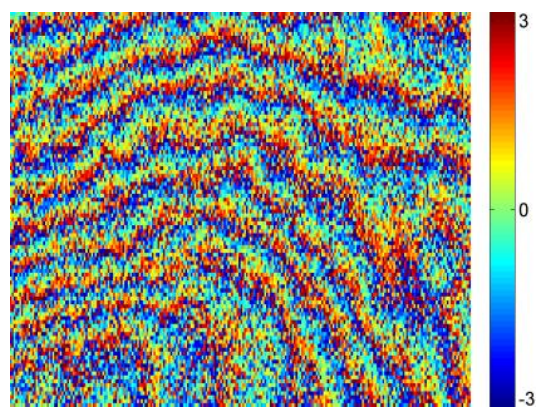

(b)

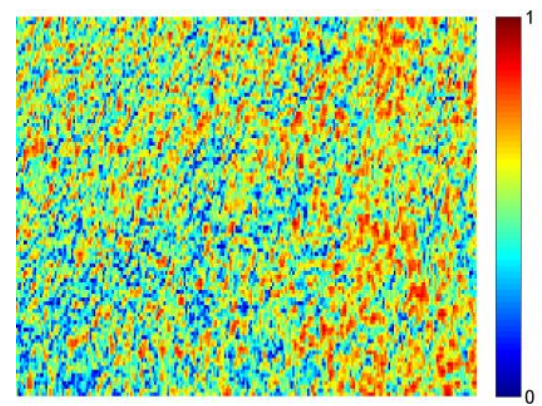

(c)

Fig. 7. Real data 1. (a) Amplitude map of master image. (b) Interferometric phase. (c) Coherence map.

The filtered phases and corresponding wrapped phase differences between the interferometric and filtered phases obtained using the PLOW, NL-InSAR and proposed methods are shown in Fig. 8. Because we do not have a noise-free phase, our analysis is mainly qualitative. As seen in Fig. 8(a), some noise still exists after filtering, which is indicative of under-filtering by the 


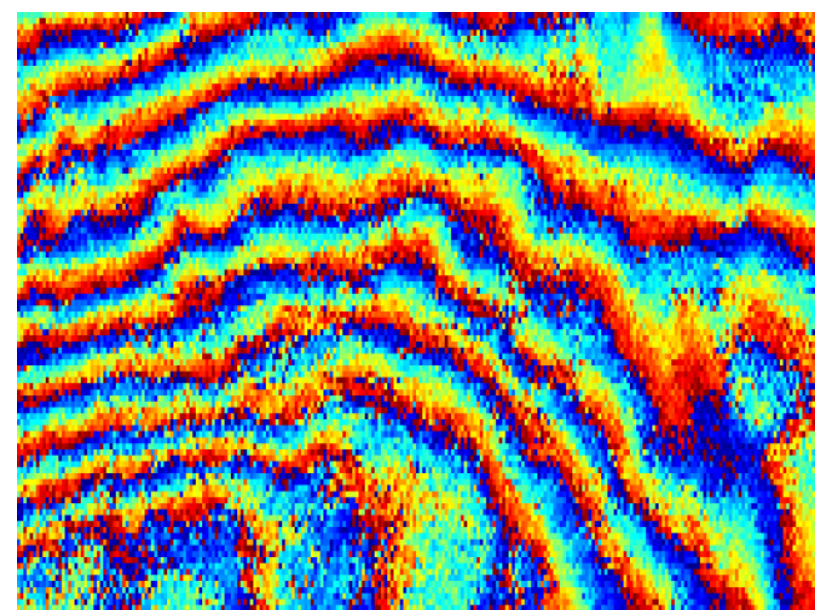

(a)

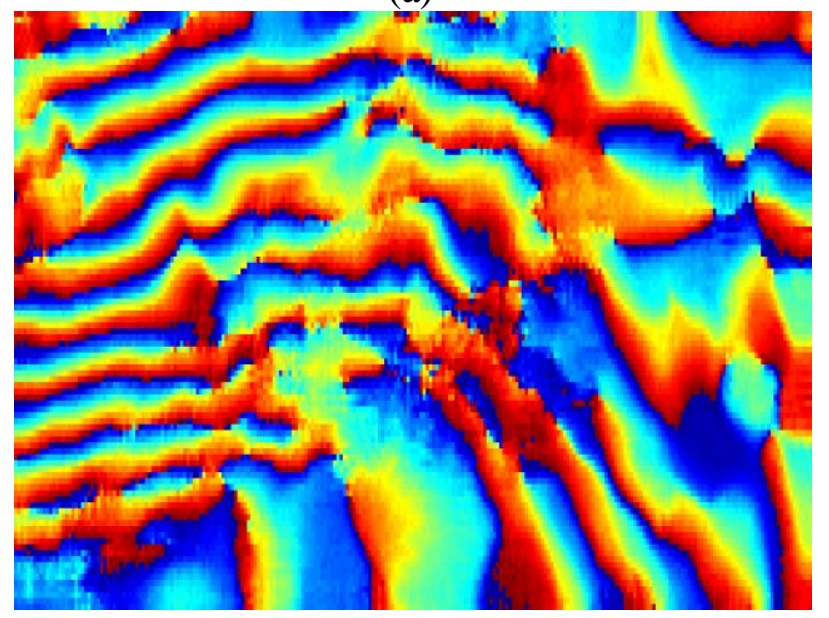

(c)

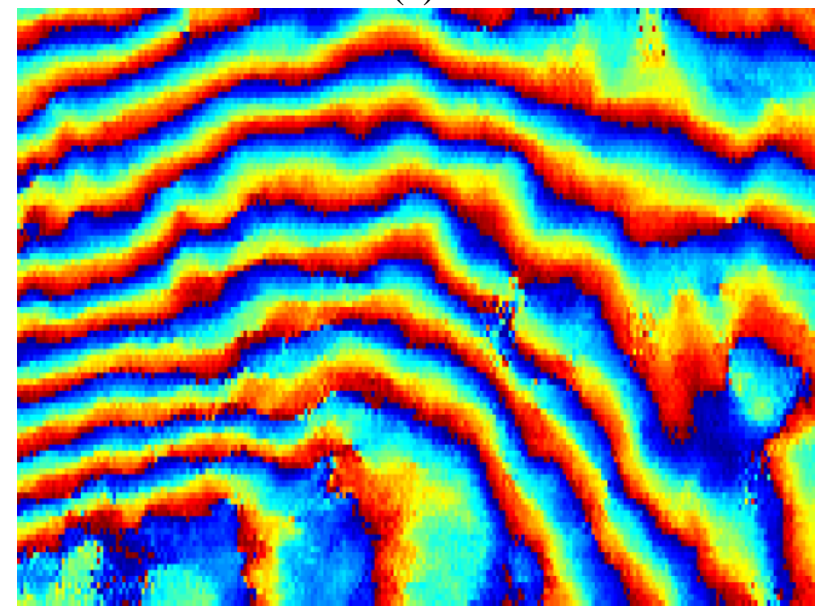

(e)

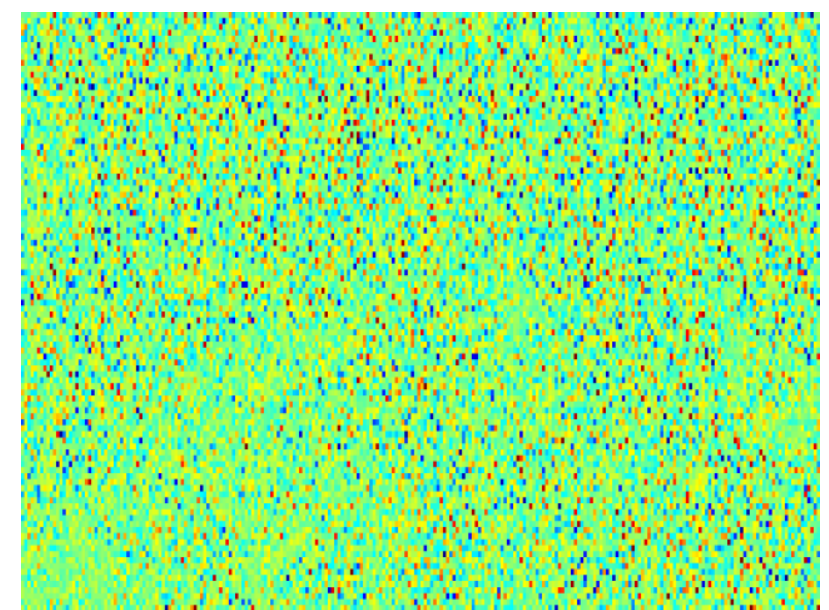

(b)

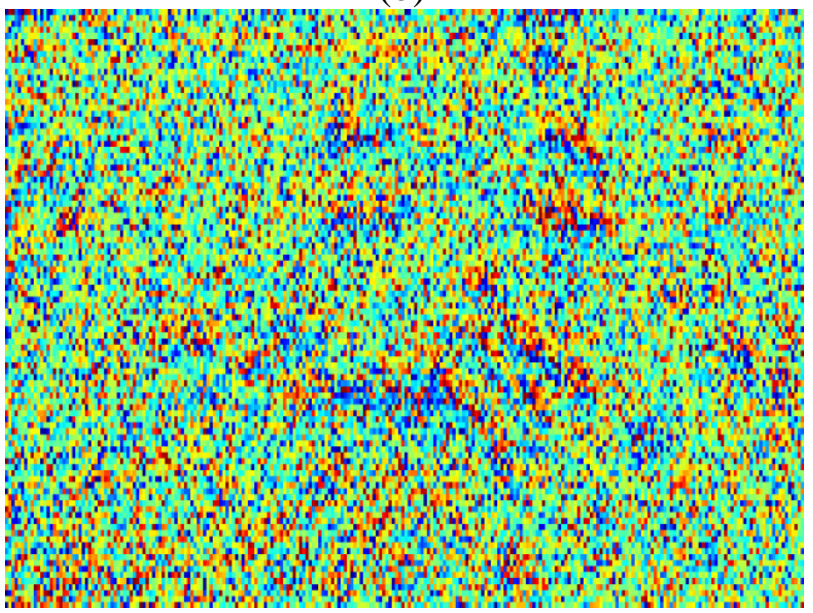

(d)

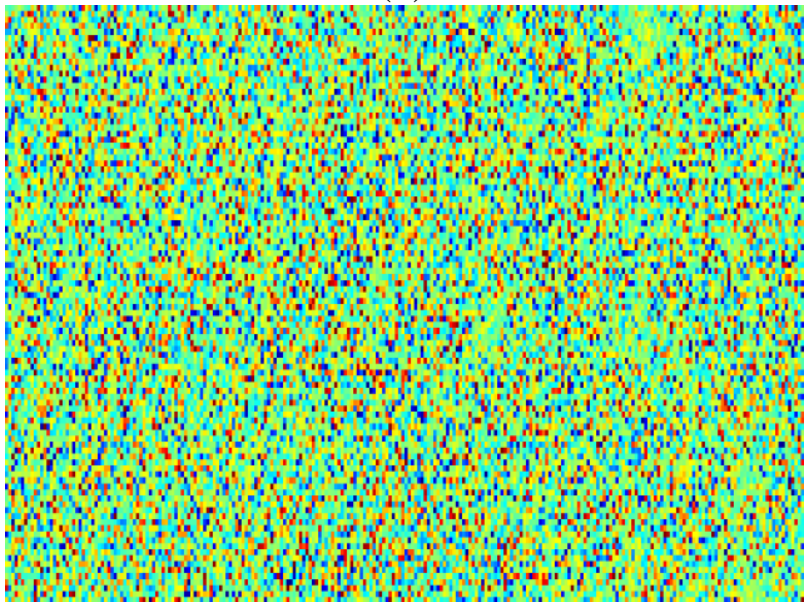

(f)

$-3$

0

3

Fig. 8. Filtered phases and wrapped phase differences. (a) Filtered phase obtained using the PLOW method and (b) its wrapped phase difference. (c) Filtered phase obtained using the NLInSAR method and (d) its wrapped phase difference. (e) Filtered phase obtained using the proposed method and (f) its wrapped phase difference. 
PLOW method. Because, according to LARK-based K-means clustering strategy, patches in one cluster may have significantly different noise levels, which cannot be properly suppressed using a global $\hat{\sigma}_{\text {median }}$. On the contrary, NL-InSAR significantly suppresses noise, but some fringes in Fig. 8(c) are broken or merged with neighbouring fringes. The proposed method yields the best balance between noise reduction and fringe preservation. To more clearly illustrate the differences between filtered phases, we display the wrapped phase difference between the interferometric and filtered phase. This difference map can represent the amount of noise that one method can remove from the interferometric phase. Besides, if a given method over-filters the phase, then structural information can also be detected in this map. Comparing Figs. 8(b), (d), and (f), one can see that the proposed method not only efficiently suppresses noise but also preserves details.

For a quantitative comparison, the three indexes were calculated and listed in Table 2. Note that the proposed method more efficiently reduces the residues and is faster than the PLOW method.

Table 2

Quantitative comparison between three methods on real data 1

\begin{tabular}{|c|c|c|c|}
\hline Method & NOR & $\begin{array}{c}\text { Percentage of } \\
\text { residue reduction } \\
(\%)\end{array}$ & $\mathrm{T}(\mathrm{s})$ \\
\hline No filtering & 5399 & - & - \\
\hline PLOW & 876 & 83.8 & 6 \\
\hline NL-InSAR & 133 & 97.5 & 34 \\
\hline Proposed & 76 & 98.6 & 2 \\
\hline
\end{tabular}




\subsection{Real data 2}

In this section, real data from ERS 1/2 tandem pair acquired over Fuji Volcano, Japan, were utilized to test the proposed method. The amplitude map of master image, interferometric phase and coherence map are shown in Figs. 9(a)-(c), respectively. From Fig. 9(c), we observe that some scattered low coherence regions exist. Those regions correspond to shadow areas, which are caused by SAR side-view imaging geometry.

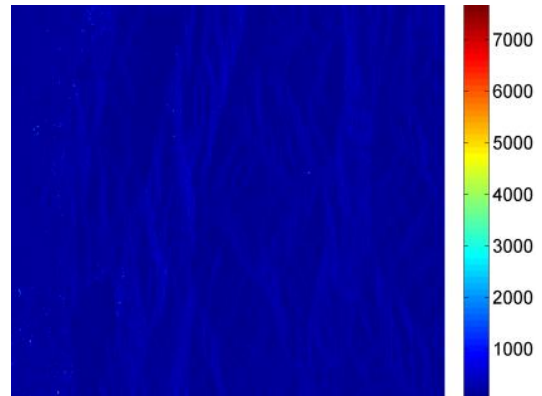

(a)

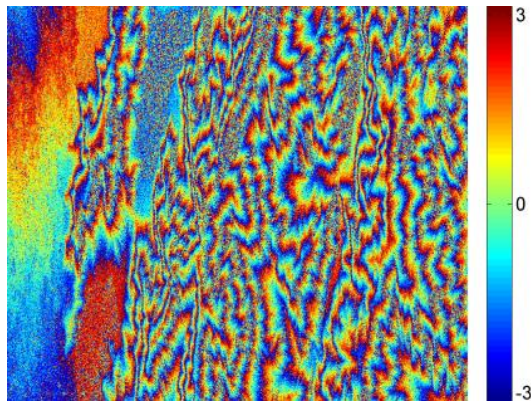

(b)

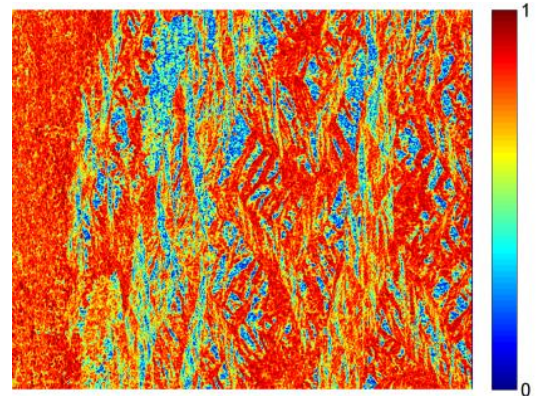

(c)

Fig. 9. Real data 2. (a) Amplitude map of master image. (b) Interferometric phase. (c) Coherence map.

Fig. 10 illustrates the filtered phases and corresponding wrapped phase differences obtained using the three methods. Via visual inspection of these results, it appears that the PLOW method filtered phase is relatively noisy not only in some flat regions but also in the regions of steep terrain. Besides, the PLOW method leaves the shadow regions almost intact as seen from Fig. 10(b). On the contrary, the NL-InSAR method removes the majority of the noise, including that in the shadow regions (Fig. 10(d)). However, some spike-like fringes are also generated by the NL-InSAR method as seen from Fig. 10(c). With three modifications, the proposed method removes more noise than the PLOW method does. Comparing Figs. 10(a) and (e) (or Figs. 10(b) and (f)), we can see that some parts of the shadow regions have been smoothed in Fig. 10(e). Because in the shadow regions, the dominant component is noise which randomly produces parts of high coherence even in those regions. Then, according to the coherence-based clustering, 


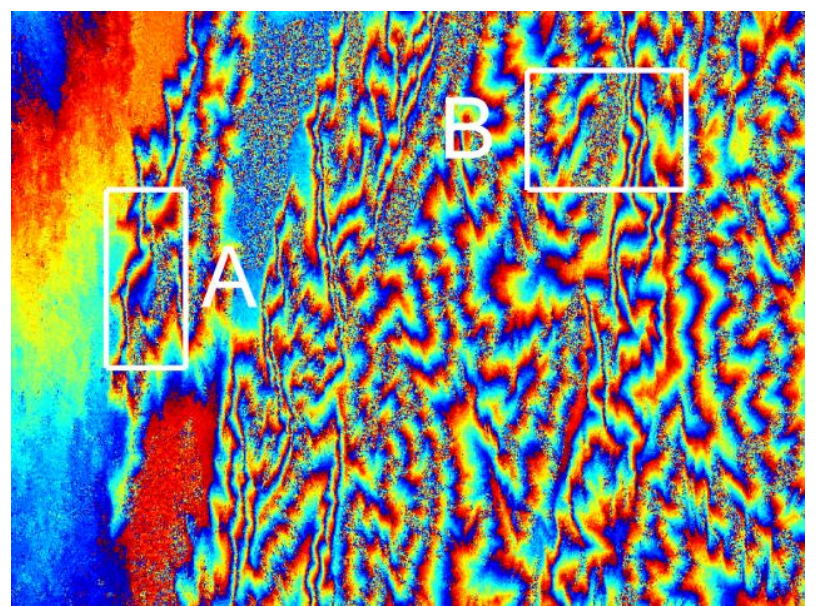

(a)

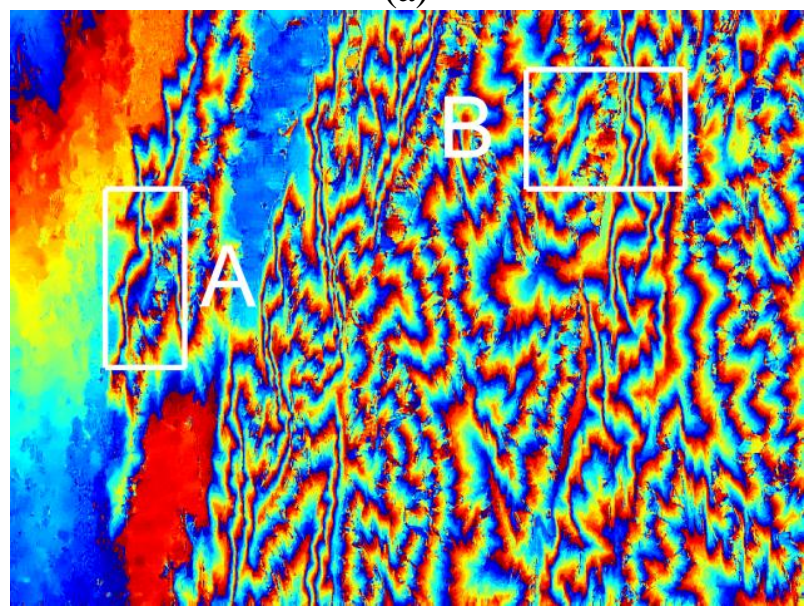

(c)

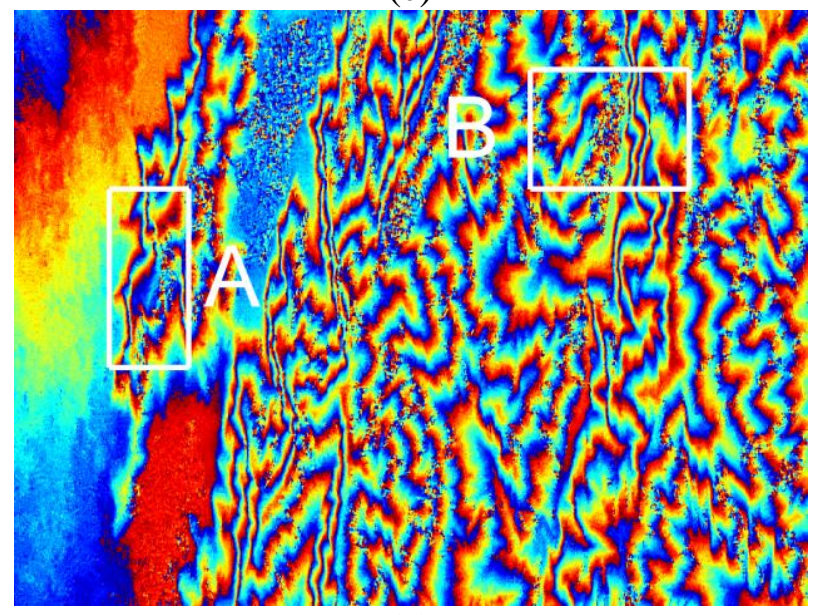

(e)

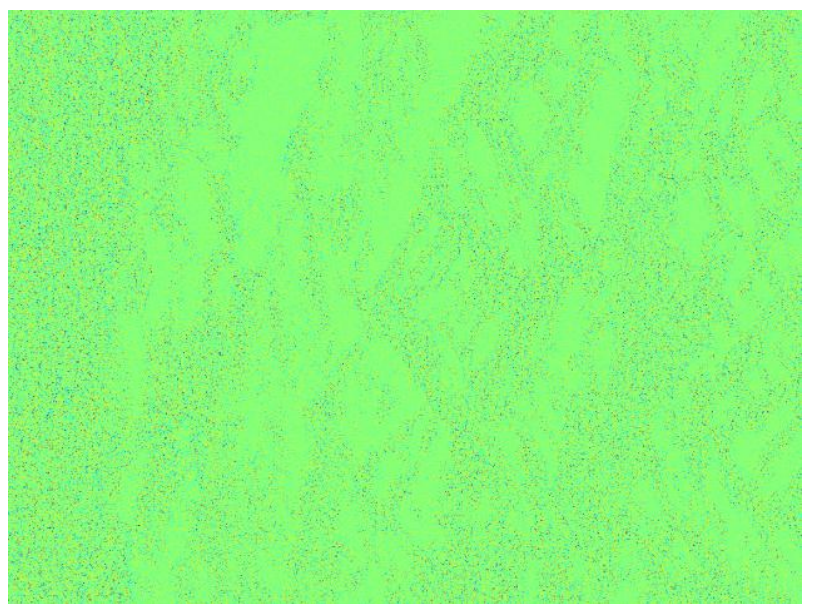

(b)

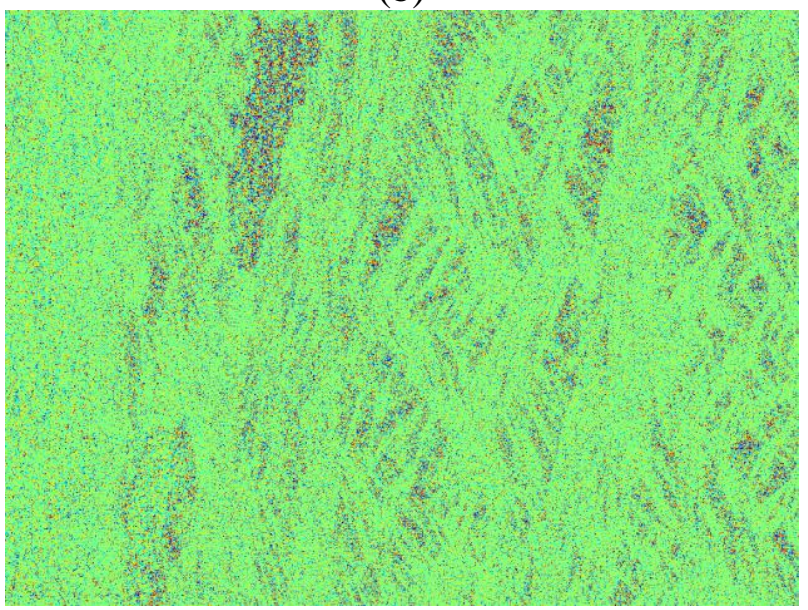

(d)

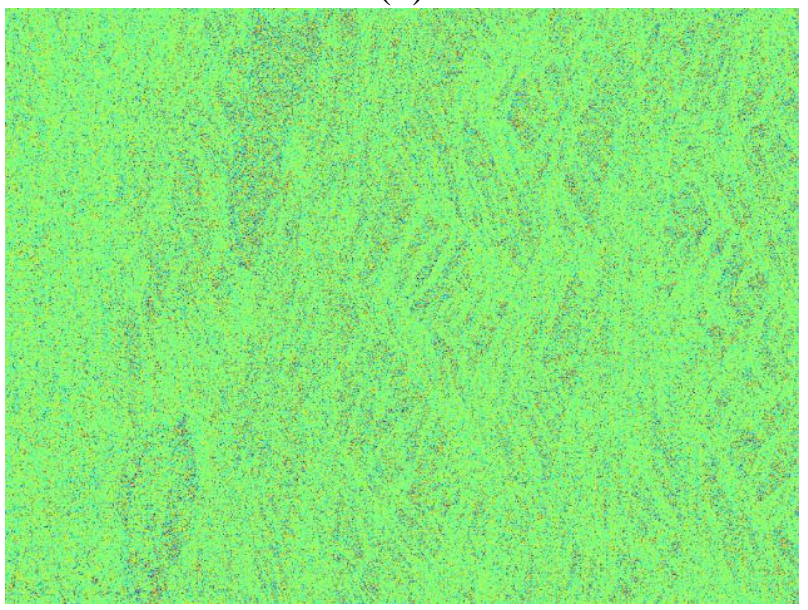

(f)

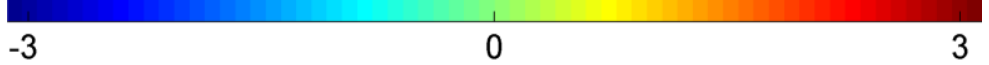

Fig. 10. Filtered phases and wrapped phase differences. (a) Filtered phase obtained using the PLOW method and (b) its wrapped phase difference. (c) Filtered phase obtained using the NLInSAR method and (d) its wrapped phase difference. (e) Filtered phase obtained using the proposed method and (f) its wrapped phase difference. 


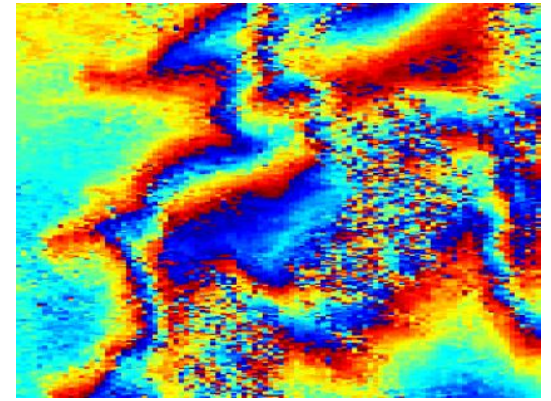

(a)

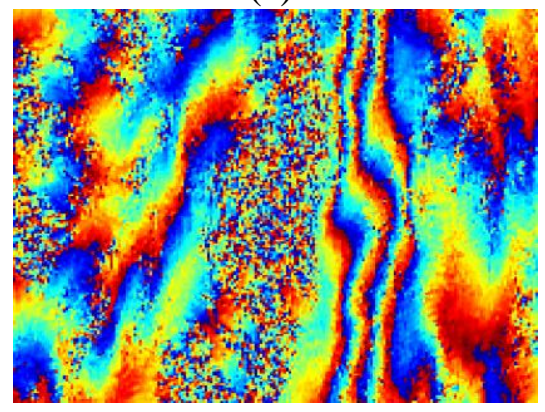

(d)

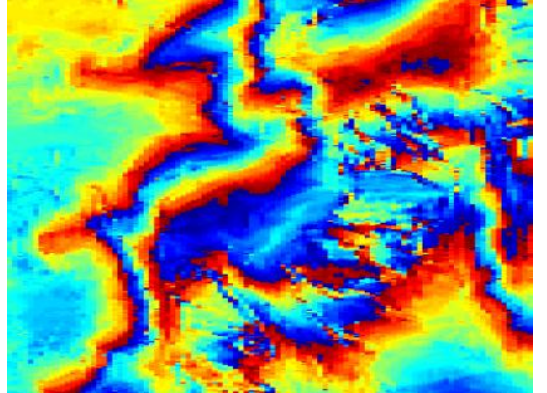

(b)

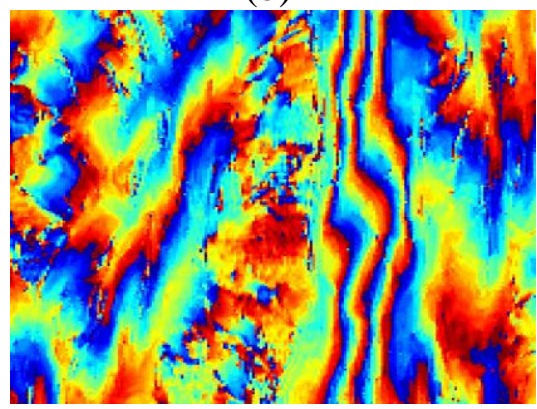

(e)

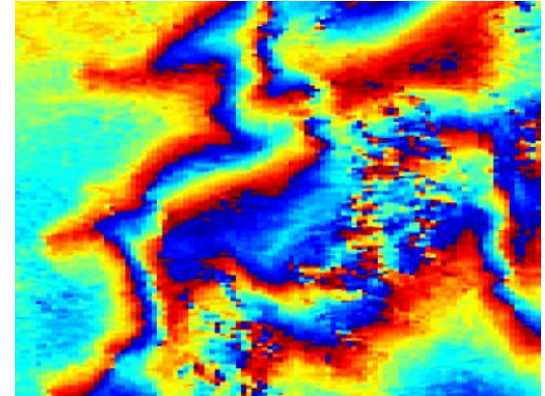

(c)

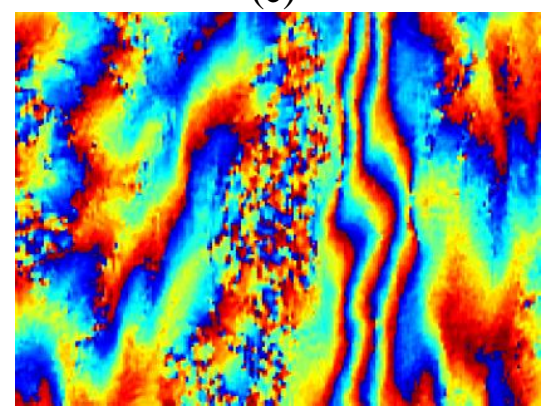

(f)

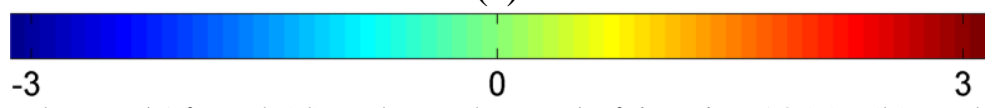

Fig. 11. (a) Enlarged area ' $A$ ' and (d) enlarged area ' $B$ ' in Fig. 10(a). (b) Enlarged area ' $A$ ' and (e) enlarged area 'B' in Fig. 10(c). (c) Enlarged area 'A' and (f) enlarged area 'B' in Fig. 10(e).

pixels from these parts are clustered together with pixels of similar coherence from non-shadow regions, thus filtered together. For clarity and detailed comparison, two areas, labelled 'A' and 'B' in Fig. 10, are enlarged in Fig. 11. From Figs. 11(a) and (d), we clearly see that noise still exists not only in the shadow regions but also in the high coherence regions. Although the fringes are smoothed in Figs. 11(b) and (e), filtering is also significant in the shadow regions, and long, thin fringes are produced in those regions. Due to strong noise, fringes recovered from shadow regions are unreliable and may induce errors in the phase unwrapping process. From Figs. 11(c) and (f), we see that the proposed method exhibits a balance between details preservation and noise reduction.

For further analysis on details preservation, the correlation coefficients (CC) between the interferometric and filtered phases are given in Table 3, together with indexes also used in Table 
2. When a filter is applied to an interferometric phase, we can also say that the phase is changed that noise is suppressed while details are inevitably removed. The degree of change could be measured by means of $\mathrm{CC}$ and the performance of noise suppression could be indicated by the NOR values, then the ability of details preservation could be inferred accordingly (roughly, change $=$ noise suppression + details removal). Comparing the PLOW and proposed methods, it is hard to tell whether the latter is better at details preservation than the former because the latter changes the phase more than the former but also reduces more noise than the former. However, it could be seen that the proposed method preserves more details than the NL-InSAR method because $\mathrm{CC}$ of the former is bigger than that of the latter and NOR of the former is smaller than that of the latter. In addition, the NL-InSAR and proposed methods eliminate more than $88 \%$ of the residues, which results in easy phase unwrapping, especially via the proposed method.

Table 3

Quantitative comparison between three methods on real data 2

\begin{tabular}{|c|c|c|c|c|}
\hline Method & CC & NOR & $\begin{array}{c}\text { Percentage of } \\
\text { residue reduction } \\
(\%)\end{array}$ & T (s) \\
\hline No filtering & 1 & 98900 & - & - \\
\hline PLOW & 0.7508 & 42050 & 57.5 & 188 \\
\hline NL-InSAR & 0.5520 & 10604 & 89.3 & 2251 \\
\hline Proposed & 0.6002 & 8826 & 91.1 & 86 \\
\hline
\end{tabular}

\section{Discussion about future work}

The NL-InSAR method is a joint estimation of the reflectivity, interferometric phase and coherence map using a weighted maximum likelihood estimator [52], with weights derived from 
intensity and interferometric phase. Then, why is the proposed method able to produce results comparable to or even better than the NL-InSAR method? The reason could be attributed to the use of coherence. From Eq. (8), one can see that the amplitude (or intensity) information is incorporated into the coherence, therefore the amplitude information is also used for filtering in the proposed method. Like the NL-InSAR and other [19] [27] methods, the PLOW method could also be extended to a joint estimation framework which is going be our future work.

Even the coherence map (estimated using Eq. (8)) used in the proposed method is biased, it does not affect the results too much because the filtering occurs patch-by-patch. This bias tolerance of patch-based method could also be found in [12] and [53] in which phase patch is filtered using the mean value of coherence magnitude of that patch. However, improvements could be obtained through an unbiased coherence estimator and this will be investigated in the future.

It is difficult to figure out a particular method (or a single index) to fully test the quality of achieved results. For noise suppression evaluation, there are many indexes. However, comparison about detail preservation is hard to make and mainly by visual inspection. The method for detail preservation evaluation given in Section 5.3 is a combination of CC and NOR. Besides, the evaluation is made both qualitatively and quantitatively. For future work, some other indexes, such as sum of phase differences (SPD) [54], could be added to make this method more proper and accurate for details preservation evaluation.

\section{Conclusions}

In this paper, we have proposed a novel method for InSAR phase filtering based on modifications to the PLOW method. Combing the PLOW framework with characteristics of the interferometric phase, we propose three modifications. First, pixels are clustered according to 
their coherence magnitudes instead of noise-robust geometric features. Then, the noise covariance is estimated within each cluster using an adaptive mean estimator. Finally, the mean of each cluster is estimated using a coherence-weighted mean. The proposed method has been validated using both simulated and real data. Experimental results consistently demonstrate the effectiveness of the proposed method in phase noise reduction, detail preservation and run time shortening.

\section{Acknowledgements}

The authors would like to thank the editors and anonymous reviewers for their valuable time and constructive suggestions that improve the quality of this paper. This work was supported by the National Science Foundation of China (no. 91438202 and 41271459) and the Aerospace Science and Technology Innovation Fund of the China Aerospace Science and Technology Corporation.

\section{References}

[1] R. Bamler and P. Hartl, "Synthetic aperture radar interferometry,” Inverse Problems, vol. 14, no. 4, R1-R54, 1998. [doi:10.1088/0266-5611/14/4/001]

[2] H. A. Zebker and J. Villasenor, "Decorrelation in interferometric radar echoes," IEEE Transactions on Geoscience and Remote Sensing, vol. 36, no. 5, pp. 950-959, 1992. [doi:10.1109/36.175330]

[3] G. Fornaro and A. M. Guarnieri, "Minimum mean square error space-varying filtering of interferometric SAR data," IEEE Transactions on Geoscience and Remote Sensing, vol. 40, no. 1, pp. 11-21, 2002. [doi:10.1109/36.981345]

[4] J.-S. Lee, K. P. Papathanassiou, T. L. Ainsworth, M. R. Grunes, and A. Reigber, "A new technique for noise filtering of SAR interferometric phase images," IEEE Transactions on Geoscience and Remote Sensing, vol. 36, no. 5, pp. 1456-1465, 1998. [doi:10.1109/36.718849] 
[5] C. F. Chao, K. S. Chen, and J.-S. Lee, "Refined filtering of interferometric phase from InSAR data," IEEE Transactions on Geoscience and Remote Sensing, vol. 51, no. 12, pp. 5313-5323. 2013. [doi: 10.1109/TGRS.2012.2234467]

[6] G. Gerraiuolo and G. Poggi, "A Bayesian filtering technique for SAR interferometric phase fields," IEEE Transactions on Image Processing, vol. 13, no. 10, pp. 1368-1378, 2004. [doi: 10.1109/TIP.2004.834661]

[7] G. Vasile, E. Trouve, J.-S. Lee, and V. Buzuloiu, "Intensity-driven adaptive-neighborhood technique for polarimetric and interferometric SAR parameters estimation," IEEE Transactions on Geoscience and Remote Sensing, vol. 44, no. 6, pp. 1609-1621, 2006. [doi:10.1109/TGRS.2005.864142]

[8] Q. F. Yu, X. Yang, S. H. Fu, X. L. Liu, and X. Y. Sun, “An adaptive contoured window filter for interferometric synthetic aperture radar," IEEE Geoscience and Remote Sensing Letters, vol. 4, no. 1, pp. 23-26, 2007. [doi:10.1109/LGRS.2006.883527]

[9] F. Baselice, G. Ferraioli, V. Pascazio, and G. Schirinzi, "Joint InSAR DEM and deformation estimation in a Bayesian framework," 2014 IEEE Geoscience and Remote Sensing Symposium (IGARSS), Quebec, Canada, pp. 398-401, 2014. [doi:10.1109/IGARSS.2014.6946442]

[10] D. Danudirdjo and A. Hirose, "InSAR image regularization and DEM error correction with fractal surface scattering model," IEEE Transactions on Geoscience and Remote Sensing, vol. 53, no. 3, pp. 1427-1439, 2015. [doi:10.1109/TGRS.2014.2341254]

[11] R. M. Goldstein and C. L. Werner, "Radar interferogram filtering for geophysical applications," Geophysical Research Letters, vol. 25, no. 21, pp. 4035-4038, 1998. [doi:10.1029/1998GL900033]

[12] I. Baran, M. P. Stewart, B. M. Kampes, Z. Perski, and P. Lilly, "A modification to the Goldstein radar interferogram filter," IEEE Transactions on Geoscience and Remote Sensing, vol. 41, no. 9, pp. 2114-2118, 2003. [doi:10.1109/TGRS.2003.817212] 
[13] Q. S. Wang, H. F. Huang, A. X. Yu, and Z. Dong, "An efficient and adaptive approach for noise filtering of SAR interferometric phase images," IEEE Geoscience and Remote Sensing Letters, vol. 8, no. 6, pp. 1140-1144, 2011. [doi:10.1109/LGRS.2011.2158289]

[14] B. Cai, D. N. Liang, and Z. D, “A new adaptive multiresolution noise-filtering approach for SAR interferometric phase image," IEEE Geoscience and Remote Sensing Letters, vol. 5, no. 2, pp. 266-270, 2008. [doi:10.1109/LGRS.2008.915942]

[15] C. L. Martínez and X. Fàbregas, "Modeling and reduction of SAR interferometric phase noise in the wavelet domain," IEEE Transactions on Geoscience and Remote Sensing, vol. 40, no. 12, pp. 2553-2566, 2002. [doi:10.1109/TGRS.2002.806997]

[16] X. J. Zha, R. S. Fu, Z. Y. Dai, and B. Liu, "Noise reduction in interferograms using the wavelet packet transform and wiener filtering”, IEEE Geoscience and Remote Sensing Letters, vol. 5, no. 3, pp. 404-408, 2008. [doi:10.1109/LGRS.2008.916066]

[17] Y. Bian and B. Mercer, "Interferometric SAR phase filtering in the wavelet domain using simultaneous detection and estimation," IEEE Transactions on Geoscience and Remote Sensing, vol. 49, no. 4, pp. 1396-1416, 2011. [doi:10.1109/TGRS.2010.2076286]

[18] W. B. Abdallah and R. Abdelfattah, "Two-dimensional wavelet algorithm for interferometric synthetic aperture radar phase filtering enhancement," Journal of Applied Remote Sensing, vol. 9, 2015. [doi:10.1117/1.JRS.9.096061]

[19] G. Xu, M. D. Xing, X. G. Xia, L. Zhang, Y. Y. Liu, and Z. Bao, "Sparse regularization of interferometric phase and amplitude for InSAR image formation based on Bayesian representation," IEEE Transactions on Geoscience and Remote Sensing, vol. 53, no.4, pp. 21232136, 2015. [doi:10.1109/TGRS.2014.2355592]

[20] A. B. Suksmono and A. Hirose, "Adaptive noise reduction for InSAR images based on a complex-valued MRF model and its application to phase unwrapping problem," IEEE Transactions on Geoscience and Remote Sensing, vol. 40, no. 3, pp. 699-709, 2002. [doi:10.1109/TGRS.2002.1000329] 
[21] L. Otmar, N. Holger, K. Stefan, and Y. Wang, "Phase unwrapping for SAR interferometry-A data fusion approach by Kalman filtering," IEEE Transactions on Geoscience and Remote Sensing, vol. 46, no. 1, pp. 47-58, 2008. [doi:10.1109/IGARSS.1999.772071]

[22] J. J. Martinez-Espla, T. Martinez-Marin, and J. M. Lopez-Sanchez, “A particle filter approach for InSAR phase filtering and unwrapping," IEEE Transactions on Geoscience and Remote Sensing, vol. 47, no. 4, pp. 1197-1211, 2009. [doi:10.1109/TGRS.2008.2008095]

[23] V. Katkovnik, J. Astola, and K. Egiazarian, "Phase local approximation (phaseLa) technique for phase unwrap from noisy data," IEEE Transactions on Geoscience and Remote Sensing, vol. 17, no. 6, 2008. [doi:10.1109/TIP.2008.916046]

[24] R. P. Chen, W. D. Yu, R. Wang, G. Liu, and Y. F. Shao, "Integrated denoising and unwrapping of InSAR phase based on markov random fields," IEEE Transactions on Geoscience and Remote Sensing, vol. 51, no. 8, pp. 4473-4485, 2013. [doi:10.1109/TGRS.2013.2268969]

[25] A. Ferretti, A. Fumagalli, F. Novali, C. Prati, F. Rocca, and A. Rucci, "A new algorithm for processing interferometric data-stacks: SqueeSAR," IEEE Transactions on Geoscience and Remote Sensing, vol. 49, no. 9, pp. 3460-3470, 2011. [doi:10.1109/TGRS.2011.2124465]

[26] A. Parizzi and R. Brcic, "Adaptive InSAR stack multi-looking exploiting amplitude statistics: A comparison between different techniques and practical results," IEEE Geoscience and Remote Sensing Letters, vol. 8, no. 3, pp. 441-445, 2011. [doi:10.1109/LGRS.2010.2083631]

[27] M. Schmitt and U. Stilla, "Adaptive multilooking of airborne single-pass multi-baseline InSAR stacks," IEEE Transactions on Geoscience and Remote Sensing, vol. 52, no. 1, pp. 305-312, 2014. [doi:10.1109/TGRS.2013.2238947]

[28] B. Pinel-Puysségur, R. Michel, and J.-P. Avouac, "Multi-link InSAR time series: Enhancement of a wrapped interferometric database," IEEE Journal of Selected Topics in Applied Earth Observations and Remote Sensing, vol. 5, no.3, 2012. [doi:10.1109/JSTARS.2012.2196758] 
[29] A. Pepe, Y. Yang, M. Manzo, and R. Lanari, "Improved EMCF-SBAS processing chain based on advanced techniques for the noise-filtering and selection of small baseline multi-look DInSAR interferograms," IEEE Transactions on Geoscience and Remote Sensing, vol. 35, no. 8, pp. 43944417, 2015. [doi:10.1109/TGRS.2015.2396875]

[30] A. Buades, B. Coll, and J.-M. Morel, “A review of denoising algorithm, with a new one,” SIAM Journal of Multiscale Modeling and Simulation, vol. 4, no. 2, pp. 490-530, 2005. [doi:10.1137/040616024]

[31] A. Buades, B. Coll, and J. M. Morel, "Nonlocal image and movie denoising," International Journal of Computer Vision, vol. 76, no. 2, pp. 123-139, 2008. [doi:10.1007/s11263-007-0052-1]

[32] P. Coupe, P. Yger, and C. Barillot, "Fast nonlocal means denoising for 3D MR images" International Conference on Medical Image Computing and Computer-Assisted Intervention, Berlin, Germany, pp. 33-40, 2006. [doi:10.1007/11866763_5]

[33] P. Coupe, P. Yger, S. Prima, P. Hellier, C. Kervrann, and C. Barillot, “An optimized blockwise nonlocal means denoising filter for 3-D magnetic resonane images" IEEE Transactions on Medical Imaging, vol. 27, no. 4, pp. 425-441, 2008. [doi:10.1109/TMI.2007.906087]

[34] T. Tasdizen, "Principal neighborhood dictionaries for nonlocal means image denoising," IEEE Transactions on Image Processing, vol. 18, no. 12, pp. 2649-2660, 2009. [doi:10.1109/TIP.2009.2028259]

[35] J. Boulanger, C. Kervrann, P. Bouthemy, P. Elbau, J.-B. Sibarita, and J. Salamero, "Patch-based nonlocal functional for denoising fluorescence microscopy image sequences," IEEE Transactions on Medical Imaging, vol. 29, no. 2, pp. 442-454, 2010. [doi:10.1109/TMI.2009.2033991]

[36] M. Maggioni, G. Boracchi, A. Foi, and K. Egiazarian, "Video denoising, deblocking and enhancement through separable 4-D nonlocal spatiotemporal transforms," IEEE Transactions on Image Processing, vol. 21, no. 9, pp. 3952-3966, 2012. [doi:10.1109/TIP.2012.2199324] 
[37] C.-A. Deledalle, L. Denis, and F. Tupin, "NL-InSAR: Non-local interferogram estimation," IEEE Transactions on Geoscience and Remote Sensing, vol. 49, no. 4, pp. 1441-1452, 2011. [doi:10.1109/TGRS.2010.2076376]

[38] X. X. Zhu, M. Lachaise, F. Adam, Y. L. Shi, M. Eineder, and R. Bamler, "Beyond the 12m TanDEM-X DEM,” 2014 IEEE Geoscience and Remote Sensing Symposium (IGARSS), Quebec, Canada, pp. 390-393, 2014. [doi:10.1109/IGARSS.2014.6946440]

[39] X. X. Zhu, R. Bamler, M. Lachaise, F. Adam, Y.L. Shi, and M. Eineder, "Improving TanDEMX DEMs by nonlocal InSAR filtering," EUSAR 2014, 10 ${ }^{\text {th }}$ European Conference on Synthetic Aperture Radar, Berlin, Germany, pp. 1-4, 2014.

[40] C.-A. Deledalle, L. Denis, F. Tupin, A. Reigber, and M. Jäger, "NL-SAR: A unified nonlocal framework for resolution-preserving (Pol)(In)SAR denoising," IEEE Transactions on Geoscience and Remote Sensing, vol. 53, no. 4, pp. 2021-2038, 2015. [doi:10.1109/TGRS.2014.2352555]

[41] R. P. Chen, W. D. Yu, R. Wang, G. Liu, and Y. F. Shao, "Interferometric phase denoising by pyramid nonlocal means filter," IEEE Geoscience and Remote Sensing Letters, vol. 10, no. 4, pp. 826-830, 2013. [doi:10.1109/LGRS.2012.2225594]

[42] J. W. Li, F. F. Li, Z. Bao, Y. L. Hu, and Z. Y. Suo, "Noise filtering of high-resolution interferograms over vegetation and urban areas with a refined nonlocal filter," IEEE Geoscience and Remote Sensing Letters, vol. 12, no. 1, pp. 77-81, 2015. [doi:10.1109/LGRS.2014.2326462]

[43] X. Lin, F. F. Li, D. D. Meng, D. H. Hu, and C. B. Ding, "Nonlocal SAR interferometric phase filtering through higher order singular value decomposition," IEEE Geoscience and Remote Sensing Letters, vol. 12, no. 4, pp. 806-810, 2015. [doi:10.1109/LGRS.2014.2362952]

[44] P. Chatterjee and P. Milanfar, "Patch-based near-optimal image denoising," IEEE Transactions on Image Processing, vol. 21, no. 4, pp. 1635-1649, 2012. [doi:10.1109/TIP.2011.2172799]

[45] H. Takeda, S. Farsiu, and P. Milanfar, "Kernel regression for image processing and reconstruction,” IEEE Transactions on Image Processing, vol. 16, no. 2, pp. 349-366, 2007. [doi:10.1109/TIP.2006.888330] 
[46] P. Chatterjee and P. Milanfar, "Clustering-based denoising with locally learner dictionaries," IEEE Transactions on Image Processing, vol. 18, no. 7, pp. 1438-1451, 2009. [doi:10.1109/TIP.2009.2018575]

[47] S. Lloyd, "Least squares quantization in PCM," IEEE Transactions on Information Theory, vol. IT-28, no. 2, pp. 129-137, 1982. [doi:10.1109/TIT.1982.1056489]

[48] D.L. Donoho and I. M. Johnstone, "Ideal spatial adaptation by wavelet shrinkage," Biometrika, vol. 81, no. 3, pp. 425-455, 1994. [doi:10.1093/biomet/81.3.425]

[49] Z. Wang, A. C. Bovik, H. R. Sheikh, and E. P. Simoncelli, "Image quality assessment: From error visibility to structural similarity," IEEE Transactions on Image Processing, vol. 13, no. 4, pp. 600-612, 2004. [doi:10.1109/TIP.2003.819861]

[50] D. C. Ghiglia and M. D. Pritt, Two-Dimensional phase unwrapping: Theory, Algorithms, and Software. New York: Wiley, 1998.

[51] B. Kampes and S. Usai, "Doris: The Delft object-oriented radar interferometric software," In Proc. ITC $2^{\text {nd }}$ ORS Symp., Netherlands, 1999, CD-ROM.

[52] M. Seymour and I. Cumming, "Maximum likelihood estimation for SAR interferometry," in The 1994 International Geoscience and Remote Sensing Symposium, vol. 4, pp. 2272-2274, 1994.

[53] M. Jiang, X. L. Ding, Z. W. Li, X. Tian, W. Zhu, C. S. Wang, and B. Xu, "The improvement for Baran phase filter derived from unbiased InSAR coherence," IEEE Transactions on Geoscience and Remote Sensing, vol. 7, no. 7, pp. 3002-3010, 2014. [doi:10.1109/JSTARS.2013.2296322]

[54] Z. L. Li, W. B. Zou, X. L. Ding, Y. Q. Chen, and G. X. Liu, "A quantitative measure for the quality of InSAR interferograms based on phase differences," Photogrammetric Engineering and Remote Sensing, vol. 70, no. 10, pp. 1131-1137, 2004. 